\title{
GPU accelerated initialization of local maximum-entropy meshfree methods for vibrational and acoustic problems
}

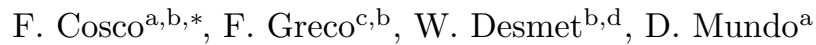 \\ ${ }^{a}$ Universitá della Calabria, DIMEG, ponte P. Bucci 45C, 87036 Rende (CS), Italy \\ ${ }^{b}$ KU Leuven, Division LMSD, Celestijnenlaan 300B - box 2420, B-3001 Leuven, Belgium \\ ${ }^{c}$ Laboratori de Calcul Numeric, Universitat Politecnica de Catalunya-BarcelonaTech, E-08034 Barcelona, Spain \\ ${ }^{d}$ DMM Lab, Flanders Make, Belgium
}

\begin{abstract}
This paper presents an efficient strategy for the matrix assembly procedure in a Galerkin implementation of local maximum-entropy (LME) meshfree schemes, using graphic processor units (GPUs) as massive parallel accelerators. LME basis functions show excellent performance in the simulation of vibrational and acoustic problems, described by the Helmholtz equation. However, even considering a locally truncated support, their evaluation requires a significantly higher number of neighbors, as compared to finite elements and other meshfree methods, which poses several challenges towards a computationally efficient allocation and filling of the required sparse matrices structures. The proposed algorithm relies on a clustering strategy, and it is structured to exploit the massive parallelism of GPU architectures. Numerical examples demonstrate that this strategy enables a substantial performance boost, deriving from a synergic effect of the relatively higher computational throughput and typically larger memory bandwidth of GPUs , as compared to conventional CPUs. For the more demanding stage, we report speedups up to $1035 \times$ when using a Titan X GPU hosted in a dedicated workstation, and a more modest yet substantial acceleration up to $91 \times$ when using a mobile workstation, finally opening up to the possibility of handling industrially relevant applications not only on dedicated high-performance computing infrastructures but also on commodity hardware.
\end{abstract}

Keywords: vibrational and acoustic analysis; maximum-entropy; meshfree; matrix assembly; GPU acceleration; CUDA; parallel computing;

\section{Introduction}

Meshfree methods, also known as meshless, have emerged over the last years as a computational alternative to the classical Finite Element Method (FEM) in the resolution of partial differential equations problems (PDEs) $[1,2,3$, 4]. As the name suggests, these methods do not rely on a mesh to construct the numerical approximation of a field variable and, as such, they posses some advantages with respect to FEM in problems involving large deformations of the computational domain. In addition, meshfree basis functions often present a high smoothness by construction.

While a detailed list of all the meshfree schemes would be quite long (see [4] for instance), we can identify the Element Free Galerkin Method (EFGM) [5] and the Reproducing Kernel Particle Method (RKPM) [6] as the first significant attempts to use a meshfree approximation for the Galerkin formulation of PDEs. These methodologies rely on the Moving Least Squares method [7] to construct the basis functions, which are in general not strictly positive and do not posses the Kronecker-delta property on the

\footnotetext{
* Corresponding author

Email address: francesco.cosco@unical.it (F. Cosco)
}

boundary of domain. Due to the lack of this property, the imposition of essential boundary conditions is not straightforward and additional efforts are needed [8].

To address these issues, other methodologies have been later developed, such as the Point Interpolation Method (PIM) [9], based on a polynomial approximation, and the Radial Point Interpolation Method (RPIM) [10] which employs radial basis functions. At the same time, other approaches have been investigated to compute meshfree approximation schemes. For instance, in the Natural Element Method (NEM) [11], the basis functions are constructed by means of geometrical entities such as the Delaunay triangulation and the Voronoi diagram of the nodes. NEM basis functions are strictly positive and interpolatory on the boundary, but their computation requires a significant effort[12].

Local maximum-entropy (LME) approximation schemes [13] represent another relevant contribution developed over the last few years. The idea of using the Jaynes's maximum entropy or max-ent principle from statistical mechanics [14] was previously introduced in [15], where the max-ent formalism appeared in the context of polygonal finite elements. LME schemes arose later on, as a seamless bridge between finite elements and meshfree methods, by combin- 
ing the entropy functional with a locality term [13]. With this modification, LME basis functions have a fast exponential decay and it is possible to numerically truncate their support.

Thanks to their formulation, LME approximants are $C^{\infty}$-continuous and strictly non-negative over the computational domain and they possess a weak Kronecker-delta property on the faces of the convex hull of the nodes: on each face, only the nodes belonging to that face have a nonzero contribution. Besides, their evaluation is performed in a robust way using duality methods, which leads to sets of non-linear equations, numerically solved by recursive schemes, such as Newton-Raphson.

Thanks to these properties, LME schemes have been successfully applied to the resolution of PDEs in many fields, such as incompressible media problems [16], metal forming and cutting simulation [17], reduced order modeling of mechanical systems [18], convection-diffusion problems [19], fracture mechanics [20], biasing of molecular simulations [21], phase-field models applied to biomembranes [22, 23], thin shell analysis [24] and time-harmonic acoustic problems [25]. More recent advances include the development of high order max-ent schemes [26, 27] and their combination with geometrically exact boundary descriptions [28, 29, 30].

Although LME basis functions have a local support, whose locality results from the truncation of the entropy functional derived shapes originally developed for the whole support, a significant amount of neighbors is obtained for each evaluation point, which demands a huge computational effort to assemble stiffness and mass matrices [31]. This is due partially to the the basis functions evaluation but more specifically to the assembly procedure of such matrices, which is a computationally demanding task for several reasons. Therefore, it can pose a severe limitation to the applicability of LME-based approaches, when their initialization phase constitutes a bottle-neck with respect to the ultimate resolution of the linear system of equations.

It is worth noting that the mentioned computational burden arises on the one hand from the relatively large support area, and on the other hand from the non homogeneous structure of the problem, as opposite to FEM where the presence of a structured mesh allows for a more regular initialization phase. Beside the increased density due to the support size, the lack of structure demands for more complex algorithms and dataflow, needed for the locally variant nature of the meshfree approach. As such, the computational flow reduces to a massively large set of very simple, quick and robust operations, making it a perfect candidate for accelerations by means of modern graphics processing units (GPU) .

In fact, driven by the growth of gaming industry and the continuous seek of better performances, GPUs and their programming models (openGL, directX) evolved over the last few years towards the programmable pipeline and become a valid alternative to the standard CPU based approach in many applications. Attracted by the tremen- dous computing performance offered by GPUs, many researchers started to maps their non-graphics problems on the available resources: it was the beginning of the General Purpose programming of GPU (GPGPU) [32]. In order to ease GPGPU programming, NVIDIA released the first version a Compute Unified Device Architecture (CUDA) framework in 2007 [33]. Beside CUDA, OpenCL ${ }^{T M}$ (open Computing Language) was introduced in 2009 as a royaltyfree standard for supporting cross-platform parallel programming of heterogenous systems. Despite its lack of portability, being focused only on NVIDIA platforms, CUDA evolved rapidly as the most mature technology with the most advanced development tools offered for GPGPU applications [34, 35].

While strong form based meshfree methods can be easily implemented on GPUs [36, 37], the adoption of GPUbased acceleration for the initialization phase of Galerkin formulations was pioneered in [38], where Karatarakis et al. originally presented a set of effective algorithms amenable for massive parallelism for the case of the EFGM. In particular, they proposed the node-pair-wise as opposed to the gauss-point-wise approach of the traditional serial algorithms. In a similar way, they also addressed the GPUbased acceleration of isogeometric analysis, presenting the interaction-wise formulation as opposed to a more traditional element-wise computation of the stiffness matrix [39].

A similar attempt was recently proposed in [31], where the typical challenges of LME schemes are effectively highlighted and solved. Due to larger influence domains, the storage of the shapes functions and their derivatives requires special attention in order to maintain spatial complexity within the feasibility boundary of commodity hardware resources. Then an element-wise approach is proposed for reducing the computational burden of computing the stiffness matrix in a sparse format.

Greatly inspired from the above mentioned works, this paper focuses on the GPU-based acceleration of the initialization phases for LME meshfree approximants and presents an efficient strategy to deal with the massive amount of required operations. In particular, we adopt the compact storage mechanism presented in [31], but also propose a new approach for the computation of mass and stiffness matrices, which relies on several levels of parallelism and makes it amenable to massive parallel accelerators, and heterogeneous computing platforms. In this approach, the domain is divided into different clusters (groups of nodes), for each of which the corresponding domain of influence is approximated efficiently by exploiting geometrical aspects. The locality of the sparsity patterns is also exploited to initialize the computational data structures required for hosting the intermediate results corresponding to a subset of rows - within a cluster, the computation flow of each row is completely independent from the others. As an application, we consider the specific case of vibrational and acoustic problems, described by the Helmholtz equation, where LME basis functions have been shown to outper- 
form standard finite element methods and to better handle dispersion errors in the high frequency regime [25]. The extension to other applications such us linear elasticity or fourth order problems is straightforward.

The remainder of the paper is organized as follows: in Section 2 the LME approximants are introduced; Section 3 focuses on the proposed initialization GPU-accelerated initialization, highlighting its typical computational challanges: a set of benchmarks is used along the section to quantify also the scalability of the proposed approach in both CPU and GPU architectures. Ultimate validation of the approach is achieved in Section 4 by considering two numerical examples. Finally, Section 5 gives some concluding remarks and outlines possible future developments.

\section{The LME approximants}

The max-ent principle was initially adopted for the computation of basis functions in the context of polygonal finite elements [15], since a correspondence was noted between basis functions and discrete probability measures. In fact, in information theory, Shannon introduced the notion of entropy as a measure of uncertainty or incomplete knowledge [40]. Then Jaynes showed that maximizing the entropy provides the least-biased statistical inference when insufficient information is available [14] and developed the maximum-entropy (max-ent) principle. If we consider a set of distinct nodes in $\Re^{d}$ that are located at $\boldsymbol{x}_{a}(a=1,2, \ldots, n)$, with $\Omega=\operatorname{con}\left(\boldsymbol{x}_{1}, \ldots, \boldsymbol{x}_{n}\right) \subset \Re^{d}$ denoting the convex hull of the nodal set, for a real-valued function $u(\boldsymbol{x}): \Omega \rightarrow \Re$, a numerical approximation for $u(\boldsymbol{x})$ is defined as

$$
u^{h}(\boldsymbol{x})=\sum_{a=1}^{n} \phi_{a}(\boldsymbol{x}) u_{a}
$$

where $\phi_{a}(\boldsymbol{x})$ are the basis functions and $u_{a}$ are the coefficients associated with the nodes. In Jaynes's max-ent formalism, an entropy functional that depends on a discrete probability measure $\left\{p_{a}\right\}_{a=1}^{n}$ is maximized, subject to linear constraints on $p_{a}$. Thus, a correspondence between basis functions $\left\{\phi_{a}\right\}_{a=1}^{n}$ and discrete probability measures $\left\{p_{a}\right\}_{a=1}^{n}$ is easily noted, since basis functions have to verify the partition of unity and the linear consistency condition.

Based on this consideration, in [15] the following variational formulation is adopted: find $\boldsymbol{x} \mapsto \boldsymbol{\phi}(\boldsymbol{x}): \Omega \rightarrow \Re_{+}^{n}$ as the solution of the following constrained (concave) optimization problem:

$$
\max _{\boldsymbol{\phi} \in \Re_{+}^{n}} H(\boldsymbol{\phi})=-\sum_{a=1}^{n} \phi_{a}(\boldsymbol{x}) \ln \phi_{a}(\boldsymbol{x}),
$$

subject to the linear reproducing conditions:

$$
\begin{aligned}
\sum_{a=1}^{n} \phi_{a}(\boldsymbol{x}) & =1, \\
\sum_{a=1}^{n} \phi_{a}(\boldsymbol{x}) \tilde{\boldsymbol{x}}_{a} & =\mathbf{0},
\end{aligned}
$$

where $\Re_{+}^{n}$ is the non-negative orthant, and $\tilde{\boldsymbol{x}}_{a}=\boldsymbol{x}_{a}-\boldsymbol{x}$ are shifted nodal coordinates with respect to node $a$.

This formulation is used in [15] to compute the shape functions of polygonal finite elements but, when applied to scattered point distributions, it presents the problem that the basis functions of each node are defined over the whole domain and, in addition, exhibit a slow decay. Therefore, in order to extend it to the context of meshfree approximation schemes, a modification was introduced in [13], such that the basis functions present a more local character and a faster decay. In particular, the max-ent variational formulation was modified by introducing a Pareto optimum between the maximization of the entropy and the locality of the basis functions. This results in the following optimization problem:

$$
\min _{\boldsymbol{\phi} \in \Re_{+}^{n}} \sum_{a=1}^{n} \beta_{a} \phi_{a}(\boldsymbol{x})\left\|\tilde{\boldsymbol{x}}_{a}\right\|^{2}+\phi_{a}(\boldsymbol{x}) \ln \left(\phi_{a}(\boldsymbol{x})\right)
$$

also subject to the constraints given by Eq. (3). The Pareto parameter $\beta_{a}$ controls the locality of the basis functions such that they become more local when $\beta$ increases until the limit case of $\beta \rightarrow \infty$ where finite element shape functions are recovered. This is proven in [41], where it is shown that the piecewise affine shape functions defined on the Delaunay triangulation of a set of nodes can be obtained through convex optimization by solving

$$
\min _{\boldsymbol{\phi} \in \Re_{+}^{n}} \sum_{a=1}^{n} \phi_{a}(\boldsymbol{x})\left\|\tilde{\boldsymbol{x}}_{a}\right\|^{2},
$$

subject to the linear constraints of Eq. (3). Since the total width is minimized, these shape functions are the most local possible functions for a given cloud of nodes. This inspired the work in [13], where by combining the two functionals, a seamless transition from purely max-ent basis functions to finite elements is obtained.

The optimization problem stated in (4) is solved using the method of Lagrange multipliers as:

$\phi_{a}(\boldsymbol{x})=\frac{Z_{a}(\boldsymbol{x} ; \boldsymbol{\lambda})}{Z(\boldsymbol{x} ; \boldsymbol{\lambda})}, \quad Z_{a}\left(\boldsymbol{x}_{a} ; \boldsymbol{\lambda}\right)=\exp \left(-\beta_{a}\left\|\tilde{\boldsymbol{x}}_{a}\right\|^{2}+\boldsymbol{\lambda} \cdot \tilde{\boldsymbol{x}}_{a}\right)$

where $\boldsymbol{\lambda}(\boldsymbol{x}) \in \Re^{d}$ are the $d$ Lagrange multipliers associated with the constraints given by Eq. $(3 \mathrm{~b})$, and $Z(\boldsymbol{x} ; \boldsymbol{\lambda})=$ $\sum_{b} Z_{b}(\boldsymbol{x} ; \boldsymbol{\lambda})$ is known as the partition function in statistical mechanics. Considering the dual formulation, a solution for the Lagrange multipliers is obtained as

$$
\boldsymbol{\lambda}^{*}=\operatorname{argmin} F(\boldsymbol{\lambda}), \quad F(\boldsymbol{\lambda}):=\ln Z(\boldsymbol{\lambda}) .
$$


According to Eq. (6), LME basis functions have theoretically a global support, defined over the domain $\Omega$. However, they decay approximately as an exponential function of the distance from their relative node and, therefore, only a limited amount of nodes in the proximity of a given evaluation point have a significant contribution to the partition function. In the numerical practice, this locality is exploited to make the evaluation feasible by defining a truncation tolerance $\mathrm{Tol}_{0}$. Then, for each node $\boldsymbol{x}_{a}$, the corresponding basis function $\phi_{a}(\boldsymbol{x})$ is truncated when the term $\exp -\left(\beta_{a}\left\|\tilde{\boldsymbol{x}}_{a}\right\|^{2}\right)$ in Eq. (6) becomes smaller than $\mathrm{Tol}_{0}$. Since $\left\|\tilde{\boldsymbol{x}}_{a}\right\|$ is the distance between an evaluation point $\boldsymbol{x}$ and $\boldsymbol{x}_{a}$, this is equivalent to consider a circular support whose radius $R_{a}$ is finite and amounts to

$$
R_{a}=\sqrt{-\log \left(\operatorname{Tol}_{0}\right) / \beta_{a}}=h \sqrt{-\log \left(\operatorname{Tol}_{0}\right) / \gamma_{a}} .
$$

On noting that the influence of $\beta_{a}$ depends on the mesh size, in the max-ent literature a non-dimensional parameter $\left\{\gamma_{a}=\beta_{a} h_{a}^{2}\right\}_{a=1, \ldots, N}$ is preferred. Typical values of $\gamma$ are in the range from 0.5 to 3 and an illustration of LME basis functions for different $\gamma$ is given in Fig. 1. As depicted in the figure, LME approximants are endowed with features such as monotonicity and $C^{\infty}$ smoothness. They also possess the variation diminishing property and satisfy the weak Kronecker-delta property on the faces of the convex hull of the nodes, which makes the imposition of essential boundary conditions in Galerkin methods straightforward. Moreover, the approximants are multidimensional and lead to well-behaved mass matrices. Remarkably, all the above mentioned advantages come with the price of an increased level of interactions between nodes and quadrature points, which makes the evaluation of the basis function and the mass and stiffness matrices assembling procedure a computationally involved task.

We refer the interested reader to [13] for a more detailed discussion about the LME basis functions evaluation and to $[42,43]$ for the computation of first and second derivatives.

\section{GPU accelerated initializations of the LME mesh- free methods}

Following the definitions given in [31], for each Gauss point $g$ its corresponding primal list, $\mathcal{P}_{g}$, includes all the nodes that contribute to its basis function: $\forall g \in \mathcal{G} \Rightarrow$ $\mathcal{P}_{g}=\left\{n \in \mathcal{N} \mid\|g-n\|<R_{g}\right\}$. Moreover, for each node $n$, its dual list, $\mathcal{D}_{n}$, is the subset of Gauss points by which the node is influenced: $\forall n \in \mathcal{N} \Rightarrow \mathcal{D}_{n}=\{g \in \mathcal{G} \mid\|g-n\|<$ $\left.R_{n}\right\}$.

Fig. 2 helps to clarify the implications due to the meshfree nature of the LME approximants, compared to FEM. The support area of LME approximants is geometrically defined, and usually much bigger than in FEM. More in particular, a given node in FEM is influenced by a very limited set of Gauss points, i.e. the ones belonging to the

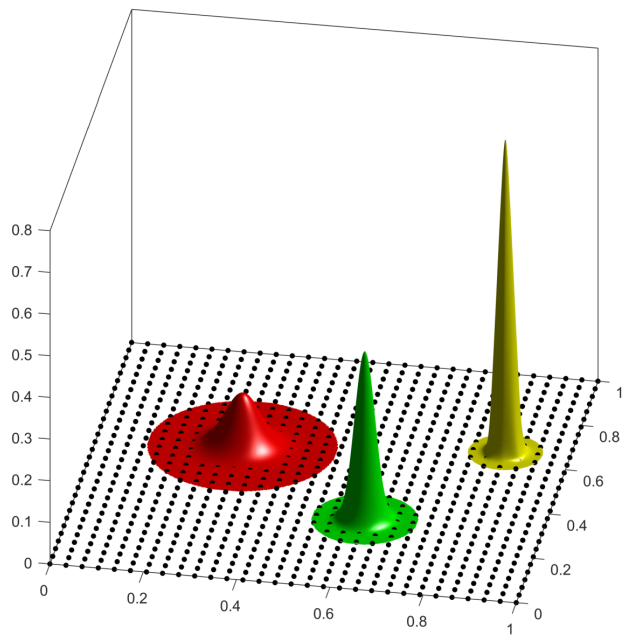

Fig. 1. LME basis functions computed on a square domain, for different values of $\gamma$. It can be observed as their shape strictly depends on $\gamma$, which in this example takes the values: $\gamma=0.63$ (red), $\gamma=1.27$ (green) and $\gamma=2.57$ (yellow). In the limit case of $\gamma \rightarrow \infty$ the Delaunay shape functions would be recovered. The size of the support depends on the truncation tolerance, which in this figure is fixed to $\mathrm{Tol}_{0}=10^{-6}$.

elements which share the node as a vertex. As a consequence, a node can interact only with a limited set of other nodes, which results in a limited number of computations. But more importantly, the resulting sparsity pattern of mass and stiffness matrices can be formulated as a function of the elements topology. On the contrary, LME Primal and Dual lists are much more populated, due to the large support sizes, and without any predefined pattern to be used for speeding up the computation. As a result,

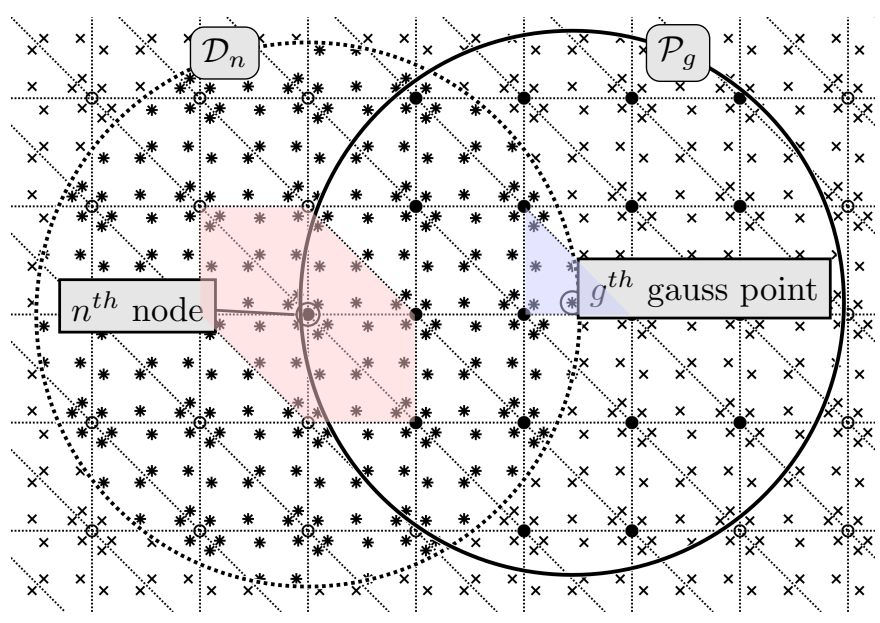

Fig. 2. Primal and dual lists defined from the nodes-Gauss points interaction. A primal list $\mathcal{P}_{g}$ collect all nodes $\bullet$ influenced by the quadrature point $g$. A dual list, $\mathcal{D}_{n}$ identifies all Gauss points * influenced by the node $n$. The Gauss points are generated using the Delaunay triangulation of the grid (dashed lines) as integration mesh. For the depicted case, the LME interpolants are generated using a support size given by Eq. 8, using Tol $_{0}=1 E-11$ and $\gamma_{a}=4.0$. As a result, the primal lists cardinality, $\left|\mathcal{P}_{n}\right|$, is bound to 20 , whereas the dual lists exhibit a cardinality 15 times larger $\left(\max \left|\mathcal{P}_{n}\right| \sim 300\right)$. 
the LME initialization phase is computationally more involved because of the increased level of interactions which brings both an higher number of computation to be executed, and a worse complexity arising from the lack of structure. Overall, higher levels of computational density can be explained as the price to be paid in order to achieve the benefits lead by the LME approximants.

Table 1 summarizes the total number of node-Gauss points correlations for a set of $2 \mathrm{D}$ problems with different mesh sizes, increasing orders of integration and different values of $\gamma$. All the examples correspond to a square domain $0.1 \times 0.1$, for which the Delaunay triangulation is used as a background integration mesh and the Dunavant rules [44] of second, forth and sixth order, corresponding respectively to 3,6 and 12 integration points are used.

In order to understand the computational impact of the increased level of interactions it is worth comparing the number of correlations of the corresponding FEM model (reported in column 4), with the corresponding value for the LME approximants, for three different values of $\gamma$ (columns 5-7). As summarized also in Fig. 3, the reported levels of interaction are dramatically higher with respect to the traditional FEM, with a relative increase ranging from 10 to 160 times larger values. It is worth noting that interactions increase whenever the support size is increased by reducing $\gamma$, or the density of Gauss points is increased by using a higher order quadrature rule.

Given the big impact of the $\gamma$ value on the computational complexity, it is worth to further analyze the geometry of the basis functions support, and its relation to $\gamma$. On the one hand, figure 2 shows as the cardinality of both primal and dual lists is strictly dependent on the local distributions of nodes and quadrature points within the domain, but also on the support size. On the other hand, according to the rigth-hand side in Eq. (8), the support radius scales proportionally to the factor under the square root, but it scales also proportionally with the mesh characteristic length, $h$.

Therefore, after normalizing the effects of $h$, we obtain:

$$
\hat{R}=\frac{R_{a}}{h}=\sqrt{\frac{-\log \left(\mathrm{Tol}_{0}\right)}{\gamma_{a}}} .
$$

The normalized support radius, $\hat{R}$, can be used for predicting the cardinality of Primal lists, as given by:

$$
\left|\mathcal{P}_{g}\right| \approx \frac{\pi}{2 d}(2 * \hat{R})^{d}
$$

where $d$ depends on the dimensionality, i.e. $d=2$ for planar problems or $d=3$ for spatial ones.

The relation expressed in Eq. (10) summarizes all the effects discussed so far, such as the influence of the shape parameter, $\gamma$, as well as of the cut-off tolerance, $\mathrm{Tol}_{0}$, on the normalized support radius and, as a consequence, on the cardinality of the Primal lists.

As depicted in Fig. 4, the same support size, thus the same density of interactions, can be obtained by dif- ferent combinations of $\gamma$ and $\mathrm{Tol}_{0}$, although the resulting LME approximation would be different. As remarked by the solid line, along the remainder of the paper we adopt a fixed value of the cut-off tolerance $\mathrm{Tol}_{0}=10^{-11}$. Moreover, by means of Eq. (10), we tune the values of $\gamma=2.57,1.27,0.63$, such to obtain a Primal list cardinality bound to at most $32,64,128$ nodes respectively, and as a consequence, increased level of interactions in the range of about 10, 20 or 40 time larger than in FEM, in agreement with the results presented in Fig. 3 and Table 1.

When solving large scale problems, coming from the discretization of PDES with a high amount of nodes, the use of the sparse formalism is needed for the storage of mass and stiffness matrices and the possibility of storing LME basis functions and their derivatives is compromised [31]. As a consequence, an effective computational strategy demands for adequate care, in order to minimize the resulting computational and spatial complexities; much more when aiming at taking advantage of the power of modern GPU for achieving massive parallelism, in the context of heterogeneous platforms.

For the developments presented along this paper, we exploit CUDA, a parallel computing platform and programming model created by NVIDIA [33], and depicted in Fig. 5. According to the CUDA programming model, work can be orchestrated to run in parallel in a hierarchical way. Different threads are simultaneously executed in warps, and multiple warps are grouped to form a block. Threads parallel execution within a block can be controlled, by means of synchronization barriers, although there is no guarantee on the order of execution. The same program is normally launched to be executed on a grid of several blocks, and there is no guarantee on the order by which different blocks will be scheduled, nor any mechanism to coordinate the execution at this level: each launch of a program is called a kernel. The host program can launch multiple kernels, whose execution is pipelined on different streams. Blocks of the various kernels, from the different pipeline are scheduled to became resident on the GPU, as soon as their resources requirements are met. Access performance to different types of memory existing on the GPU, such as registers, caches, shared memory, constant memory, depends on the execution hierarchy, with performances generally decreasing moving further than the thread level. More information on the CUDA programming model are given in [45, 38, 39].

All the performance results showed along the remainder of the section have been executed on a workstation featuring a Intel(R)Xeon(R)10 cores E5-2640v4 CPU with 2400-3400(boost) MHz clock speed, $64 \mathrm{~GB}$ of $2400 \mathrm{MHz}$ DDR4 RDIMM ECC memory, and a NVIDIA TITAN X Pascal card featuring 3584 CUDA cores, running at 14181531(boost) MHz 12 GB of GDDR5X memory, with a total bandwitdh of $480 \mathrm{~GB} / \mathrm{s}$. In particular, in this work, we compare three different $\mathrm{C}++$ implementations of the same algorithm: 


\section{Table 1}

Node-Gauss points interactions in FEM and LME for different values of the aspect ratio parameter $\gamma$.

The order of integration $i$, and the number of nodes along each side of the domain, $n$, are indicated within each example labels, according to the syntax En.i

\begin{tabular}{lcccccc}
\hline Example & Nodes & Gauss pts. & FEM & \multicolumn{3}{c}{ LME Schemes } \\
& & & & $\gamma=2.57$ & $\gamma=1.27$ & $\gamma=0.63$ \\
\hline$E 101.2$ & 10201 & $6.0 E+04$ & $1.8 E+05$ & $1.8 E+06$ & $3.7 E+06$ & $7.3 E+06$ \\
$E 101.4$ & 10201 & $1.2 E+05$ & $3.6 E+05$ & $3.6 E+06$ & $7.3 E+06$ & $1.4 E+07$ \\
$E 101.6$ & 10201 & $2.4 E+05$ & $7.2 E+05$ & $7.5 E+06$ & $1.5 E+07$ & $2.9 E+07$ \\
$E 201.2$ & 40401 & $2.4 E+05$ & $7.2 E+05$ & $7.2 E+06$ & $1.5 E+07$ & $3.0 E+07$ \\
$E 201.4$ & 40401 & $4.8 E+05$ & $1.4 E+06$ & $1.5 E+07$ & $3.0 E+07$ & $5.9 E+07$ \\
$E 201.6$ & 40401 & $9.6 E+05$ & $2.9 E+06$ & $3.0 E+07$ & $5.9 E+07$ & $1.2 E+08$ \\
$E 401.2$ & 160801 & $9.6 E+05$ & $2.9 E+06$ & $2.9 E+07$ & $6.0 E+07$ & $1.2 E+08$ \\
$E 401.4$ & 160801 & $1.9 E+06$ & $5.8 E+06$ & $6.0 E+07$ & $1.2 E+08$ & $2.4 E+08$ \\
$E 401.6$ & 160801 & $3.8 E+06$ & $1.2 E+07$ & $1.2 E+08$ & $2.4 E+08$ & $4.8 E+08$ \\
$E 801.2$ & 641601 & $3.8 E+06$ & $1.2 E+07$ & $1.1 E+08$ & $2.4 E+08$ & $4.8 E+08$ \\
$E 801.4$ & 641601 & $7.7 E+06$ & $2.3 E+07$ & $2.4 E+08$ & $4.7 E+08$ & $9.6 E+08$ \\
$E 801.6$ & 641601 & $1.5 E+07$ & $4.6 E+07$ & $4.7 E+08$ & $9.5 E+08$ & $1.9 E+09$ \\
\hline
\end{tabular}

- $\mathbf{C P U} \mathbf{S T}_{\mathbf{S T}}$, used as a common reference, contains the single-thread implementations. Profiling required for setting affinity of the CPU to use only one logical core, and higher-priority.

- $\mathbf{C P U _ { \mathbf { M T } }}$, refers to the multi-threaded implementation. The number of parallel threads is fixed to the amount of available logic processors, which correspond to twice the number of cores.

- GPU, regards the profiling of the corresponding implementations with CUDA 10. Profiling measures listed in the paper are obtained on the CPU hosting program, thus comprise the time required for eventually migrating data back and forth from the device.

All the profiling execution times were obtained using the high_resolution_clock timers available from the chrono standard library since $\mathrm{C}++11$.

As depicted in Fig. 6, and in agreement with the state of the art, the proposed algorithm for the efficient initialization of LME approximants in a meshfree context consists of four stages: Clustering, Nears Computing, Shapes Computing and matrices Computing. The remainder of the section describes the details of each stage, discussing parallel implementations and their relative performance.

\subsection{Clustering}

Similarly to [38], the parallel algorithm presented in this work relies on regioning because this positively affects all the following stages. However, instead of focusing only on the quadrature points, we split also the nodes, into different regions of space, which are referred to as clusters. The splitting is an efficient operation, which is realized in $\mathcal{O}(n)$, as it requires two loops over the set to be split: the first for sizing the bins, and the second to fill them according to the collected size.

As depicted in Fig. 7, for a given cluster $i$, we define the list of nodes belonging to it, $\mathcal{C N}_{i}$, as wells as the clustered dual lists, $\mathcal{C D}_{i}$, comprising all the Gauss points within support distance at the frontier of the cluster, and the clustered Primal lists, $\mathcal{C} \mathcal{P}_{i}$, which comprise all the nodes within support distance from the frontier of $\mathcal{C D}_{i}$, thus potentially interacting with the nodes of the cluster.

Computation of $\mathcal{C P}{ }_{i}$ and $\mathcal{C D}_{i}$ is achieved by means of a geometrically-driven heuristic consisting of the following steps:

1. select the largest value of support size for the cluster, $R_{h}$, which is a function of the characteristic length, $h$;

2. (a) compute the Minkowski sum given by the frontier of the cluster and a circle of radius $R_{h}$ : all Gauss point belonging to it define $\mathcal{C D}_{i}$;

(b) compute the Minkowski sum given by the frontier of the cluster and a circle of radius $2 R_{h}$ : all nodes belonging to it define $\mathcal{C} \mathcal{P}_{i}$.

We note that computing $\mathcal{C P}$, with an over-estimated support radius will result in a collection with more nodes than necessary. In any case, given a certain node of a cluster, a small set of nodes of the corresponding clustered Primal list will not be in interaction with it. This approach corresponds to a relaxation on the ambition of computing the exact interaction pattern. It results in overestimating the interactions, with the approximation worsening with increasing sizes of the clusters. However, this approach allows for a very efficient reduction of the node-pair in- 

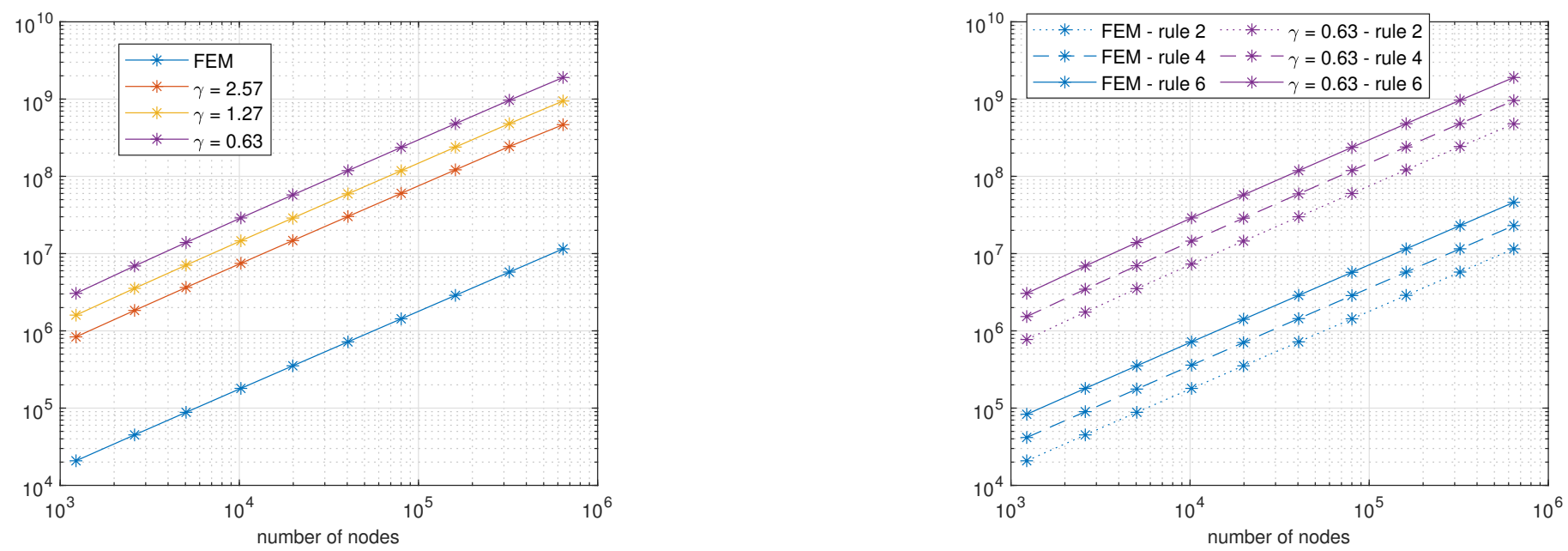

Fig. 3. Left: Number of nodes-Gauss points interactions as a function of the shape parameter $\gamma$, with a quadrature rule $=6$. The corresponsing FEM (rule=2) is reported as reference. Right: Number of nodes-Gauss points interactions as a function of the quadrature rules for both LME $(\gamma=0.63)$ and FEM are reported.

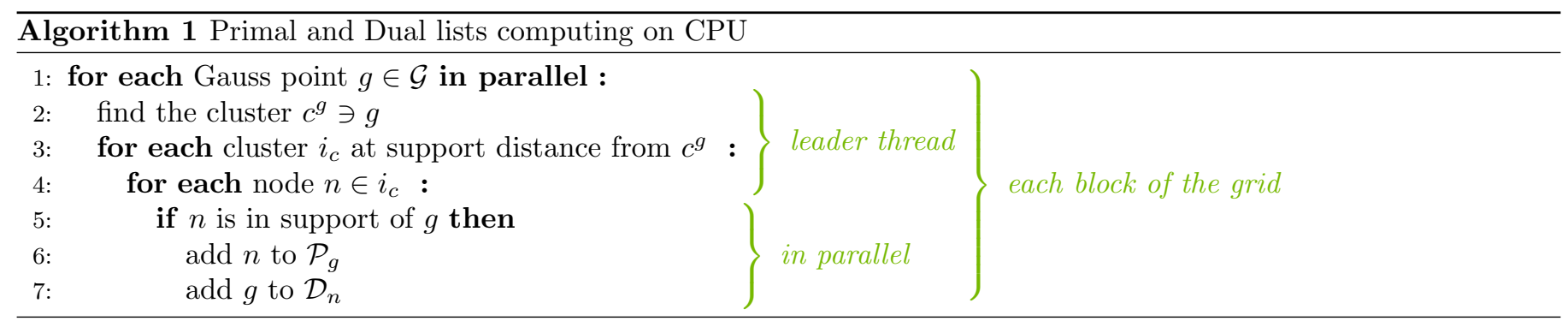

teractions search, without the need of actually computing $\mathcal{C D}$.

\subsection{Nears Computing}

The retrieval of both Primal and Dual lists falls in the class of the neighbor finding problems. The simpler solution relies on looking for any possible pair $(\mathcal{O}(|\mathcal{N}||\mathcal{G}|)$, whereas more advanced schemes exists, such as the approximate nearest neighbor searching algorithm, achieving less than quadratic performances. [31, 46]. However, thanks to

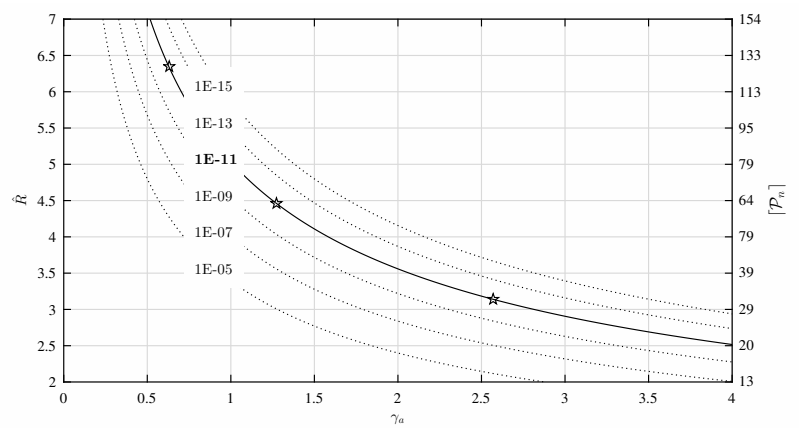

Fig. 4. A Trend of the normalized support radius $\hat{R}$, with respect to the non-dimensional parameter $\gamma_{a}$ for several values of the truncation tolerance, $\mathrm{Tol}_{0}$. Right axis shows the estimated cardinality of the primal lists by means of Eq. (8) for the planar case. the clustering approach, we are able to achieve much better performances, because for each cluster of nodes, and

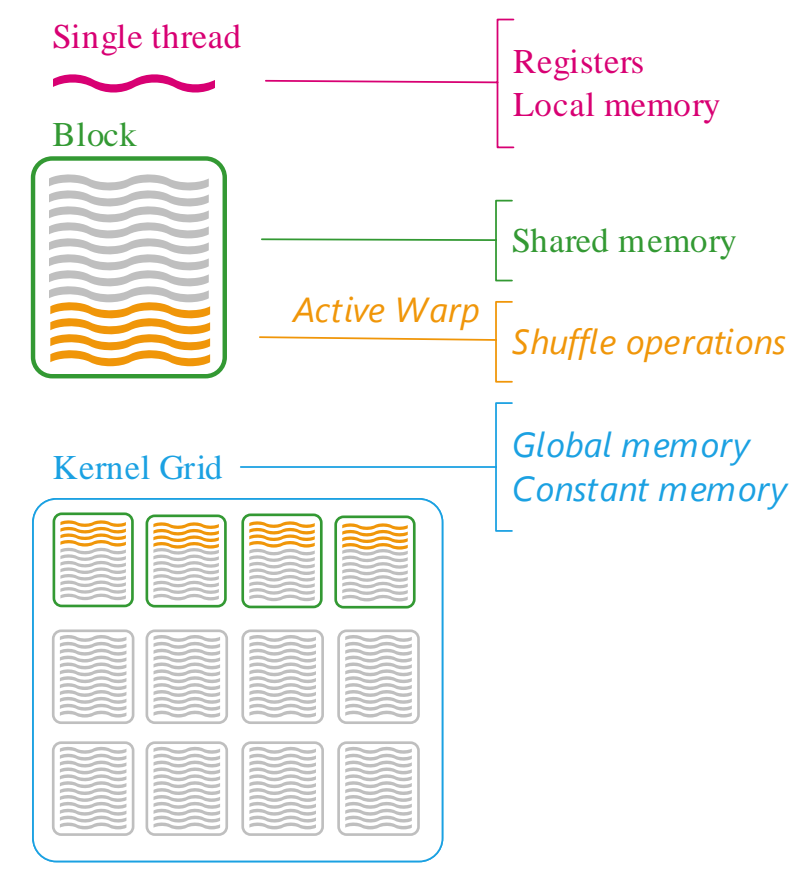

Fig. 5. Cuda Programming Model: thread and memory hierarchies. 


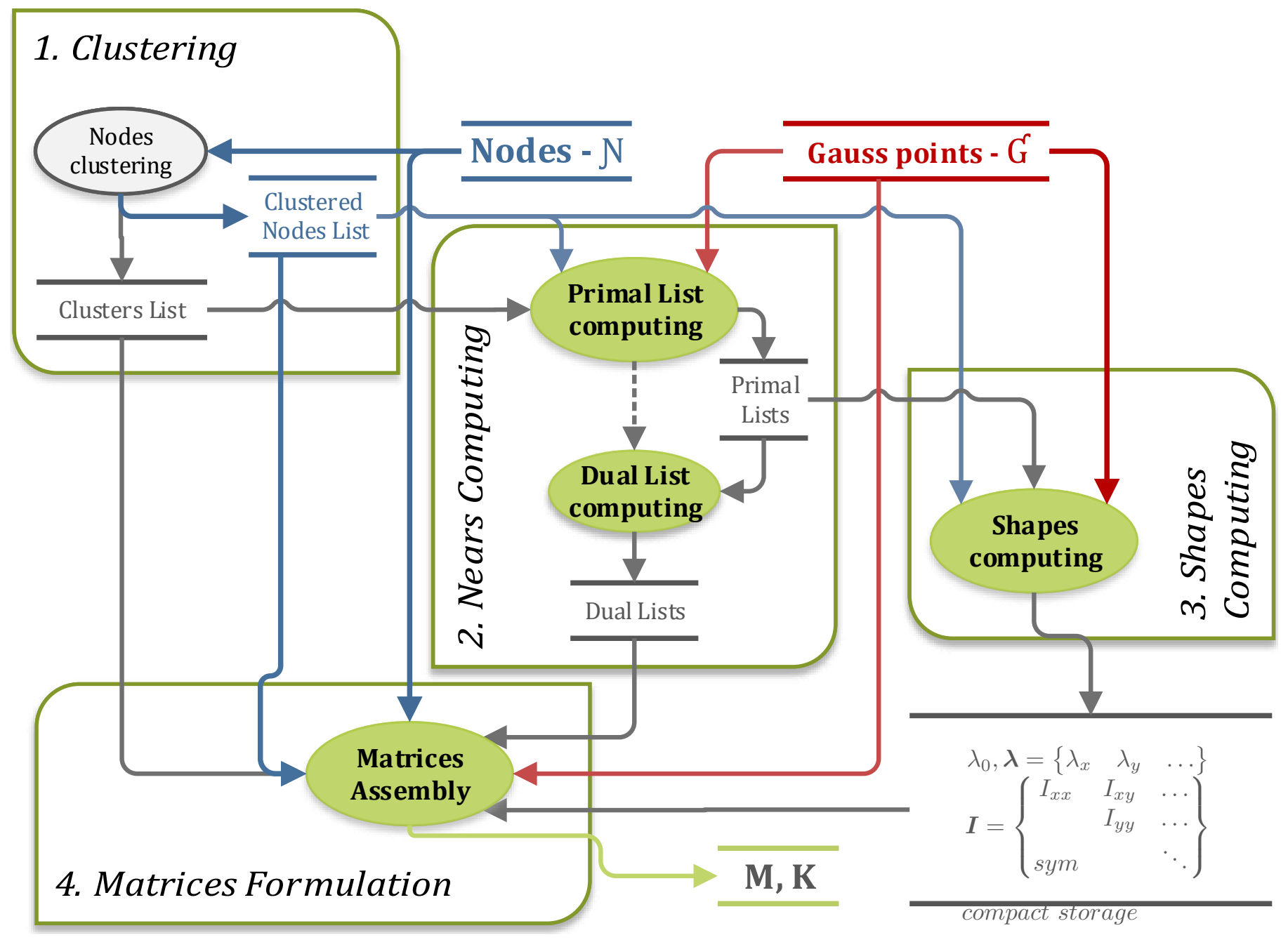

Fig. 6. Data flow of the proposed for the formulation of the mass and stiffness matrices. The process flow consists in four stages, each of which is described along Section 3.

its average support radius, the number of checks is limited only to the Gauss points belonging to those clusters at support size distance. This results in a substantial reduction with respect to the mentioned approaches, with the computational complexity being linearly proportional to the actual amount of interactions.

The proposed approach is listed more in detail in Algorythm 1. Parallel execution on CPU is achieved by splitting the jobs at the level of the outer loop at line 1. Each paralel thread is responsible of one quadrature point, and most of the work is parallel except of the race conditions that may occur at line 7 where multiple threads may try to add concurrently their Gauss point, $g$, to the same Dual list, $\mathcal{D}_{n}$. In order to increase the efficiency, Primal and Dual lists are stored using dynamically sized vectors: this allows both lists to be computed in a single stage.

For achieving the same goals on GPU, we use two kernels. The Primal lists are stored in a dedicated data structure consisting in a matrix with $N_{g}=|\mathcal{G}|$ columns and the number of rows sized according to Eq. (10), and a vector with $N_{g}$ elements. The references of each Primal list are stored in each column of the matrix, whereas the vector is used to store their cardinality. Both kernels are launched with the grid size mapping the outer loop, and block size given by Eq. (10), in order to have enough threads to run in parallel the inner loop (lines:4-9), with only one leader thread per block to orchestrate the advancing of the middle levels loops.

To avoid race conditions operations at lines $6-7$, we exploit atomic operations: regarding line 6 , a shared counter is atomically incremented by each thread of the block and the resulting incremented locations are used for directing the concurrent storage of the nodes, $n$, into the Primal list, $\mathcal{P}_{g}$. Similarly, for line 7 , the cardinality of the corresponding Dual list is atomically incremented. Although atomic operations drastically limit the performance of massively parallel executions, the described strategy benefits from two facts: the paradigm used to increment the local index is generally known as privatization, and it allows to exploits the very short latency of atomic operations on a 


\section{Table 2}

Computing time in seconds required for retrieving all node-Gauss point correlations. Speedups, relative to $C P U_{S T}$, are reported in parenthesis.

\begin{tabular}{|c|c|c|c|c|c|c|c|c|c|}
\hline Example & $C P U_{S T}$ & $\begin{array}{c}\gamma=2.57 \\
C P U_{M T}\end{array}$ & $G P U$ & $C P U_{S T}$ & $\begin{array}{c}\gamma=1.27 \\
C P U_{M T}\end{array}$ & $G P U$ & $C P U_{S T}$ & $\begin{array}{l}\gamma=0.63 \\
C P U_{M T}\end{array}$ & $G P U$ \\
\hline$E 101.2$ & 0.2 & $0.04(4.1 x)$ & $0.01(29 x)$ & 0.3 & $0.04(6.1 x)$ & $0.01(46 x)$ & 0.5 & $0.08(6.0 x)$ & $0.01(79 x)$ \\
\hline E101.4 & 0.4 & $0.07(5.1 x)$ & $0.01(50 x)$ & 0.5 & $0.10(5.3 x)$ & $0.01(78 x)$ & 0.9 & $0.21(4.3 x)$ & $0.01(115 x)$ \\
\hline E101.6 & 0.7 & $0.20(3.4 x)$ & $0.01(77 x)$ & 1.1 & $0.21(5.1 x)$ & $0.01(135 x)$ & 1.8 & $0.33(5.3 x)$ & $0.01(198 x)$ \\
\hline E201.2 & 0.7 & $0.13(5.4 x)$ & $0.01(89 x)$ & 1.1 & $0.18(6.3 x)$ & $0.01(162 x)$ & 2.0 & $0.28(7.1 x)$ & $0.01(197 x)$ \\
\hline$E 201.4$ & 1.4 & $0.30(4.8 x)$ & $0.01(159 x)$ & 2.3 & $0.41(5.5 x)$ & $0.01(173 x)$ & 3.8 & $0.59(6.4 x)$ & $0.01(253 x)$ \\
\hline$E 201.6$ & 2.8 & $0.51(5.5 x)$ & $0.02(166 x)$ & 4.4 & $0.71(6.3 x)$ & $0.02(262 x)$ & 7.3 & $1.00(7.3 x)$ & $0.03(262 x)$ \\
\hline E401.2 & 2.9 & $0.52(5.5 x)$ & $0.02(179 x)$ & 4.6 & $0.73(6.2 x)$ & $0.02(269 x)$ & 8.0 & $1.16(6.9 x)$ & $0.03(276 x)$ \\
\hline E401.4 & 5.8 & $0.95(6.1 x)$ & $0.03(200 x)$ & 9.1 & $1.30(7.0 x)$ & $0.04(239 x)$ & 15.4 & $2.10(7.3 x)$ & $0.05(296 x)$ \\
\hline E401.6 & 11.4 & $1.74(6.6 x)$ & $0.07(166 x)$ & 17.9 & $2.58(7.0 x)$ & $0.07(264 x)$ & 30.2 & $3.99(7.6 x)$ & $0.10(318 x)$ \\
\hline$E 801.2$ & 11.4 & $1.82(6.3 x)$ & $0.06(178 x)$ & 18.3 & $2.54(7.2 x)$ & $0.07(266 x)$ & 30.4 & $3.99(7.6 x)$ & $0.09(323 x)$ \\
\hline$E 801.4$ & 23.9 & $3.40(7.0 x)$ & $0.13(190 x)$ & 35.1 & $4.84(7.3 x)$ & $0.12(283 x)$ & 61.2 & $7.83(7.8 x)$ & $0.17(366 x)$ \\
\hline$E 801.6$ & 44.9 & $6.45(7.0 x)$ & $0.24(184 x)$ & 70.0 & $9.52(7.4 x)$ & $0.24(296 x)$ & 118.8 & $14.92(8.0 x)$ & $0.31(388 x)$ \\
\hline
\end{tabular}

shared variable instead of the corresponding global memory atomic intrinsic, which are notably worse. Besides, given the particular structure of the problem, the atomic increments result in a very low number of threads being serialized, because chances that the same Gauss-point-node pair is checked concurrently are low. The second kernel deals with computing the Dual lists and relies again on a privatization strategy with a shared indexing variable incremented in order to assign a private writing location to the threads aiming at writing to the same list concurrently.

In practice, the first kernel populates the Primal lists

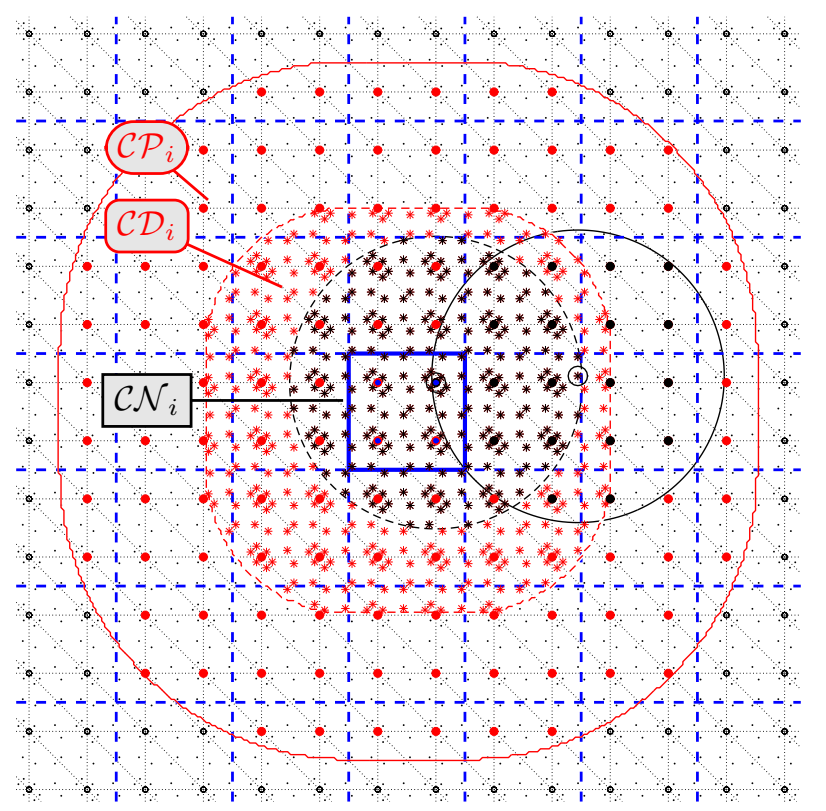

Fig. 7. Clustering. After dividing the domain in clusters (dashed blue lines), for a given cluster (full blue square in the middle), the task is to define the following lists: the cluster nodes set $\mathcal{C N}_{i}$, the clustered Primal list $\mathcal{C P}$, and the clustered dual list $\mathcal{C} \mathcal{D}_{i}$. (line 6) but also accumulates the cardinality of the corrsepnding Duals lists. Once it ends, we use the computed cardinality to allocate the Dual List data structure. The second kernel is launched afterwards, on a lower priority stream, similar to the one discussed, but focusing on filling the Dual Lists. As depicted in Fig. 8-top, the low-priority second kernel is executed only after the computation phase of the shapes has completed. Considering that the Dual list computation is suitable for CPU, our final implementation relies on the hybrid computing approach depicted in Fig. 8-bottom, where the CPU is in charge of computing the dual lists, while the GPU focus on the more demanding task of computing the shapes.

Table 2 summarizes the performance of the discussed implementations, comparing a single-threaded execution, as well as the multi-threaded execution on CPU, and the

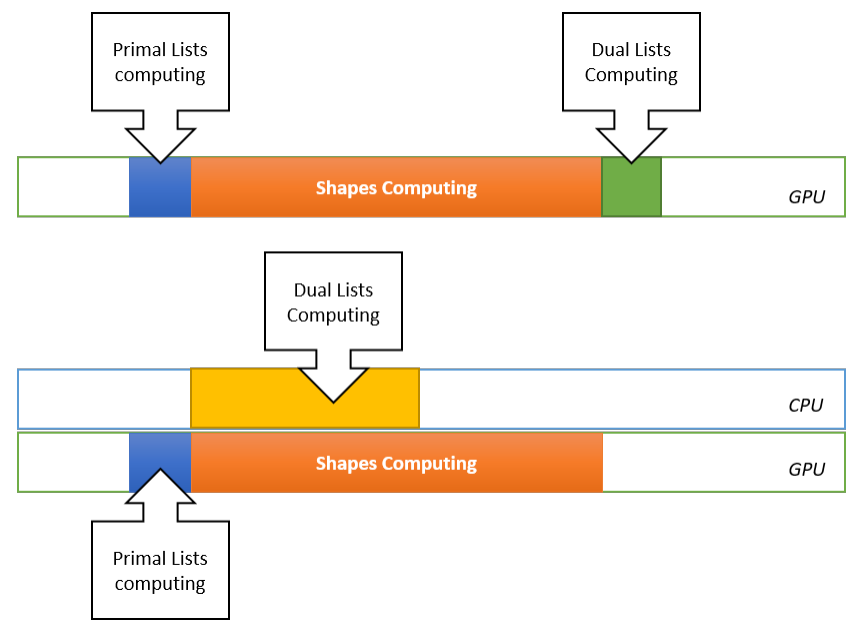

Fig. 8. Top: Primal and dual lists are computed launching two different kernels, with the dual lists computed right after the shapes. Bottom: dual lists are computed using CPU, while the GPU is kept busy on retrieving the shape functions 
Table 3

Shapes functions computing time in seconds. Speedups, relative to $C P U_{S T}$, are reported in parenthesis.

\begin{tabular}{|c|c|c|c|c|c|c|c|c|c|}
\hline Example & $C P U_{S T}$ & $\begin{array}{l}\gamma=2.57 \\
C P U_{M T}\end{array}$ & $G P U$ & $C P U_{S T}$ & $\begin{array}{l}\gamma=1.27 \\
C P U_{M T}\end{array}$ & $G P U$ & $C P U_{S T}$ & $\begin{array}{l}\gamma=0.63 \\
C P U_{M T}\end{array}$ & $G P U$ \\
\hline$E 101.2$ & 0.17 & $0.02(8.3 x)$ & $0.005(35 \mathrm{x})$ & 0.26 & $0.03(8.7 x)$ & $0.008(33 \mathrm{x})$ & 0.35 & $0.04(8.4 x)$ & $0.012(29 \mathrm{x})$ \\
\hline$E 101.4$ & 0.35 & $0.04(8.3 x)$ & $0.012(30 \mathrm{x})$ & 0.51 & $0.06(8.3 x)$ & $0.017(30 \mathrm{x})$ & 0.70 & $0.09(8.1 x)$ & $0.021(33 x)$ \\
\hline$E 101.6$ & 0.73 & $0.08(9.0 x)$ & $0.026(28 \mathrm{x})$ & 1.02 & $0.11(8.9 x)$ & $0.033(31 \mathrm{x})$ & 1.38 & $0.14(9.9 x)$ & $0.043(32 \mathrm{x})$ \\
\hline$E 201.2$ & 0.70 & $0.08(8.3 x)$ & $0.021(33 x)$ & 1.04 & $0.11(9.7 x)$ & $0.033(31 \mathrm{x})$ & 1.38 & $0.14(9.8 x)$ & $0.041(34 \mathrm{x})$ \\
\hline E201.4 & 1.45 & $0.17(8.6 x)$ & $0.040(36 \mathrm{x})$ & 2.04 & $0.21(9.6 x)$ & $0.065(31 \mathrm{x})$ & 2.74 & $0.26(10.4 x)$ & $0.074(37 x)$ \\
\hline E201.6 & 2.91 & $0.29(10.2 x)$ & $0.081(36 \mathrm{x})$ & 4.09 & $0.39(10.4 x)$ & $0.115(36 \mathrm{x})$ & 5.42 & $0.57(9.6 x)$ & $0.157(35 \mathrm{x})$ \\
\hline$E 401.2$ & 2.81 & $0.31(9.0 x)$ & $0.076(37 x)$ & 4.09 & $0.41(9.9 x)$ & $0.101(40 \mathrm{x})$ & 5.46 & $0.56(9.7 x)$ & $0.158(35 \mathrm{x})$ \\
\hline E401.4 & 5.80 & $0.57(10.3 x)$ & $0.152(38 \mathrm{x})$ & 8.21 & $0.78(10.5 x)$ & $0.236(35 \mathrm{x})$ & 10.9 & $1.02(10.6 x)$ & $0.291(37 \mathrm{x})$ \\
\hline$E 401.6$ & 11.6 & $1.05(11.1 x)$ & $0.339(34 \mathrm{x})$ & 16.5 & $1.52(10.8 x)$ & $0.448(37 x)$ & 21.7 & $1.97(11.1 x)$ & $0.521(42 \mathrm{x})$ \\
\hline$E 801.2$ & 11.0 & $1.04(10.7 x)$ & $0.348(32 \mathrm{x})$ & 16.5 & $1.53(10.8 x)$ & $0.442(37 \mathrm{x})$ & 21.4 & $1.93(11.1 x)$ & $0.516(42 \mathrm{x})$ \\
\hline$E 801.4$ & 23.0 & $2.08(11.0 x)$ & $0.630(36 x)$ & 32.5 & $2.83(11.5 x)$ & $0.793(41 x)$ & 43.2 & $3.78(11.4 x)$ & $0.983(44 x)$ \\
\hline$E 801.6$ & 45.0 & $3.97(11.4 x)$ & $1.224(37 \mathrm{x})$ & 65.0 & $5.63(11.6 x)$ & $1.564(42 \mathrm{x})$ & 85.6 & $7.39(11.6 x)$ & $1.926(44 \mathrm{x})$ \\
\hline
\end{tabular}

GPU version of the same task. As shown also in Fig. 9, the proposed approach scales well on both CPU and GPU. As expected, GPU perfomances seem to saturate for very big sizes. The shape parameter $\gamma$ plays also an important role, with some performance penalty paid when it is below $\gamma=1.27$. Regarding the CPUs, we observe a a similar trend with $C P U_{M T}$ speedups ranging between $6.4 \times$ and $8.5 \times$, with performances increasing with bigger sizes of the problem. The saturation of the speedups below $10 \times$ is due to the already mentioned impact of atomic operations, which tends to be better tolerated for larger size grids of points, where chances of atomic conflicts reduce.

\subsection{Basis functions evaluation}

For each quadrature point, the basis functions are computed by solving the optimization problem stated by Eq. 7 . This is achieved by means of duality methods with the Lagrange multipliers, as listed in Algorithm 2. At convergence, the basis functions and their spatial derivatives are

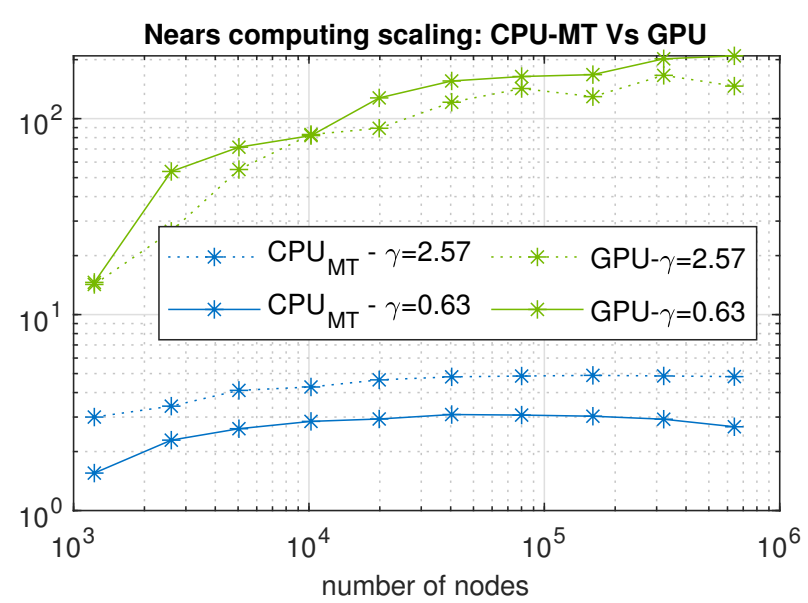

Fig. 9. Nears Computing: CPU-parallel Vs GPU speedup for different levels of $\gamma$. computed as:

$$
\begin{aligned}
\phi_{n}^{g} & =\lambda_{0}^{g} \exp \left[-\left(\beta_{g} \tilde{\boldsymbol{x}}_{g}^{n}+\boldsymbol{\lambda}^{\boldsymbol{g}}\right)^{T} \tilde{\boldsymbol{x}}_{g}^{n}\right] \\
\nabla \phi_{n}^{g} & =\left\{\phi_{n}^{g} \boldsymbol{I}^{g} \tilde{\boldsymbol{x}}_{g}^{n}\right\} .
\end{aligned}
$$

Parallel execution on CPU is achieved by splitting the work of the outer loop over several threads - as much as allowed from the hardware - such that each thread contributes to different quadrature points, thus avoiding any risk of race conditions. In the GPU version, a kernel is launched, with as many blocks as quadrature points, and block dimension regulated again using Eq. (10). In particular, each thread in a block takes care of one of the nodes in the support. However, parallelism is not perfect as the implementation requires several reduction sum operations, and relies on parts of the loop to be executed sequentially (only by the leader thread), thus we require to introduce several synchronization barriers. Besides the mentioned divergence issues, the proposed algorithm scales well on

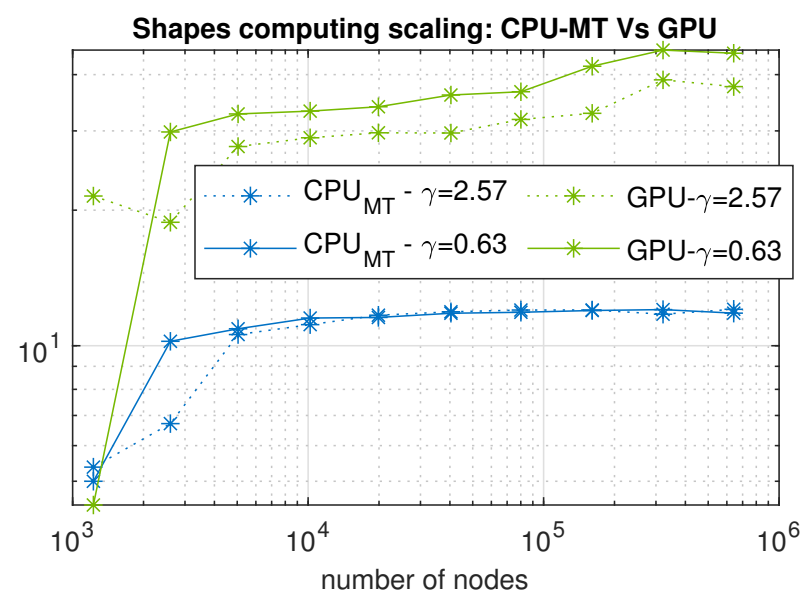

Fig. 10. Shapes Computing: $C P U_{M T}$ and $G P U$ speedup, relative to $C P U_{S T}$. 
both CPU and GPU, achieving a speed-up of around $12 \mathrm{x}$ and 40x, respectively. Table 3 summarizes the different performances of the three versions of the proposed implementations. As depicted in Fig. 10, the reported speed-up of the CPU-MT version is uniform and saturates around a value of $12 x$, whereas the GPU offers a slowly increasing speed-up value, which saturates above $40 \mathrm{x}$.

Due to the relatively higher cardinality of Primal lists, storing the basis functions and their derivatives becomes problematic when the size of the problem increases. To overcome this issue, it is worth noting that the evaluation of eq. (11) can be performed starting from the values of $\lambda_{0}^{g}, \boldsymbol{\lambda}^{g}, \boldsymbol{I}^{g}$. Therefore, as noted in [31], a compact storage strategy can be defined, by saving only such values at each evaluation point. In particular, given a certain number of quadrature points $N_{g}$ and assuming an average cardinality of Primal lists of $\overline{N_{p}}$, for the bi-dimensional case the full storage would consume $\mathcal{O}\left(3 \overline{N_{p}} N_{g}\right)$ floating point numbers, whereas the compact storage strategy would reduce the memory footprint to $\mathcal{O}\left(6 N_{g}\right)$, thus improving spatial complexity by a factor of $0.5 \overline{N_{p}}$. For the three-dimensional case we would have respectively $\mathcal{O}\left(4 \overline{N_{p}} N_{g}\right)$ for the full storage, and $\mathcal{O}\left(10 N_{g}\right)$ for the compact, obtaining a slightly lower gain of $0.4 \overline{N_{p}}$. Thus, for aspect ratios ranging between 0.5 and 4.0 , compact storage would use between $77 \mathrm{x}$ and $10 \mathrm{x}$ times less space than the full storage.

\subsection{Mass and stiffness matrix computation}

This work considers the assembling procedure of mass and stiffness matrices for problems described by the Helmholtz equation. Such equation is used to model many physical problems and two specific numerical examples corresponding to the vibrations of a membrane and to the acoustic wave propagation are considered more in detail in Section

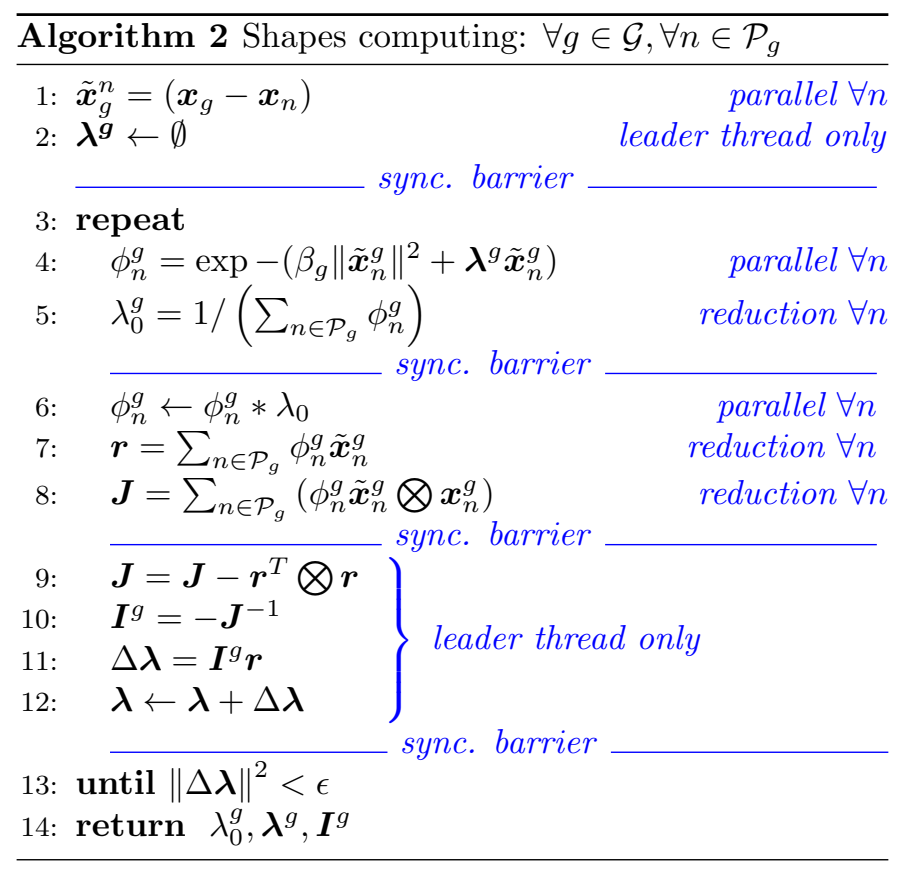

4. The homogeneous second-order Helmholtz equation is given by

$$
\nabla^{2} u+k^{2} u=0
$$

where $u$ is the unknown field and $k=\omega / c$ is the wavenumber, obtained as the ratio between the pulsation, $\omega$, and the speed of sound, c. Applying the Galerkin method, a set of algebraic equations is obtained in the form [47]

$$
\left(\mathbf{K}-\omega^{2} \mathbf{M}\right) \mathbf{d}=\mathbf{0},
$$

where $\mathbf{M}$ and $\mathbf{K}$ are the mass and stiffness matrices respectively and $\mathbf{d}$ contains the unknown basis functions coefficients [48]. The formulation of these matrices requires the summation of all the fundamental contributions, $m_{i j}^{g}$ and $k_{i j}^{g}$, due to a quadrature point, $g$, shared by a pair of nodes, $i$ and $j$, both belonging to its support area:

$$
\begin{aligned}
& \mathbf{M}=\left\{m_{i j}=\sum_{g \in \mathcal{D}_{i} \cap \mathcal{D}_{j}} m_{i j}^{g}\right\} \\
& \mathbf{K}=\left\{k_{i j}=\sum_{g \in \mathcal{D}_{i} \cap \mathcal{D}_{j}} k_{i j}^{g}\right\} .
\end{aligned}
$$

For the specific case of the Helmholtz problem, these contributions are given by the following:

$$
\begin{aligned}
m_{i j}^{g} & =\frac{1}{c^{2}} \phi_{i}^{g} \phi_{j}^{g} w_{g} \\
k_{i j}^{g} & =\nabla \phi_{i}^{g \top} \nabla \phi_{j}^{g} w_{g},
\end{aligned}
$$

where $w_{g}$ is the Gauss weight associated to each quadrature point. As a consequence, the computation of the mass and stiffness matrices can be formulated in several flavors, depending on the order by which the summation of all fundamental contributions is executed: algorithm 3 summarize a few possible alternatives.

Given the exponential growth of the spatial complexity related to the storage of the matrices, also indicated in table 4 , the usage of a sparse formalism is not only required by the limited memory resources typical of commodity hardware, but also accessory to unleash the efficiency of dedicated linear algebra packages. Coordinate list (COO) formats have been adopted in [38]: three lists are used for storing respectively the row and columns indexes as well as the values, with the size of each list equal to the number of non-zero element within the matrix. More spatially

\section{Table 4}

Full storage requirements for different size of $2 \mathrm{D}$ grids, using a 32 bit floating number representation.

\begin{tabular}{c|cc}
\hline & Nodes & Full Matrix fp32 [GB] \\
\hline E101 & 10201 & 0.4 \\
E201 & 40401 & 6.1 \\
E401 & 160801 & 96 \\
E801 & 641601 & 1533 \\
\hline
\end{tabular}


Algorithm 3 Mass and Stiffness matrix formulation for the Helmholtz problem

Gauss point-wise:

1: $M \leftarrow \emptyset, K \leftarrow \emptyset$

2: for all $g \in \mathcal{G}$ :

for all $i \in \mathcal{P}_{g}$ :

for all $j \in \mathcal{P}_{g}$ :

$M_{i j} \leftarrow M_{i j}+m_{i j}^{g}$

$K_{i j} \leftarrow K_{i j}+k_{i j}^{g}$
Node pair-wise:

1: $M \leftarrow \emptyset, K \leftarrow \emptyset$

2: for all $i \in \mathcal{N}$ :

3: for all $j \in \mathcal{N}:$

4: $\quad$ for all $g \in \mathcal{D}_{i} \cap \mathcal{D}_{j}$ :

5: $\quad M_{i j} \leftarrow M_{i j}+m_{i j}^{g}$

6: $\quad K_{i j} \leftarrow K_{i j}+k_{i j}^{g}$
Interactions-wise:

1: $M \leftarrow \emptyset, K \leftarrow \emptyset$

2: for all $i \in \mathcal{N}$ :

3: for all $g \in \mathcal{D}_{i}$ :

4: $\quad$ for all $j \in \mathcal{P}_{g}$ :

$5:$

$6:$

$$
\begin{aligned}
& M_{i j} \leftarrow M_{i j}+m_{i j}^{g} \\
& K_{i j} \leftarrow K_{i j}+k_{i j}^{g}
\end{aligned}
$$

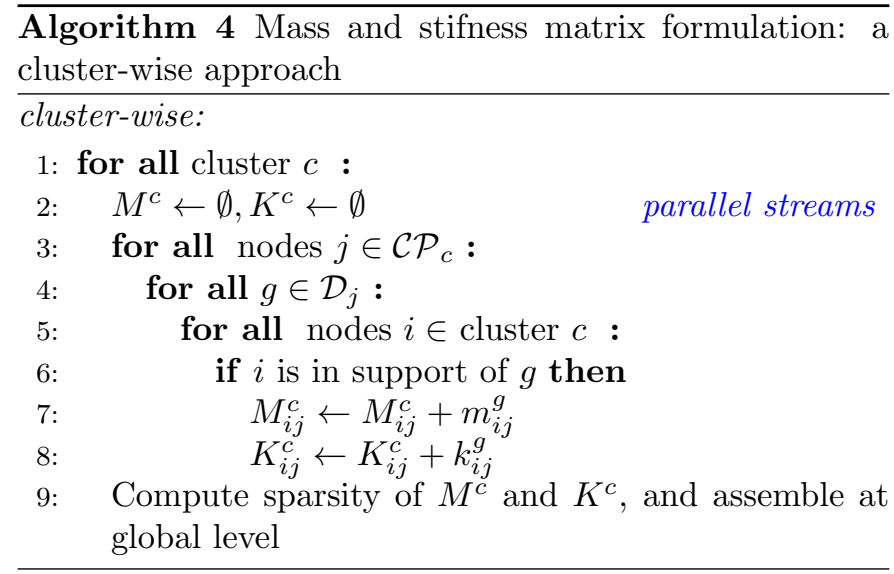

efficient alternative have also been proposed, such as the compressed sparse row (CSR) format used in [31] or the compressed sparse column (CSC), which reduces the size of one of the three lists at the expense of extra computations required when accessing the matrices during operations.

Adversely, the skyline matrix storage (SKS) has been largely adopted within FEM codes for structural mechanics applications: it consists in storing, for each row, only the block of element between the first and last non-zero entry. Dedicated SKS implementation relying on dictionary of keys, or hash-tables, can be specialized for mitigating the burden due to updating the non-zero elements a large number of times: such an implementation has been proposed in [38] for accelerating the Gauss-point wise approach. In fact, the use of simpler storage formats, such as $\mathrm{COO}$ or CSR, would require to split the matrices formulation in two stages, one for computing the sparsity pattern and the other for updating the non-zero entries.

Peco et al. [31] adopted the CSR format, and proposed to alleviate the matrix computations by considering a cell/element-wise approach. In particular, they divided the domain in cells, each one collecting a set of Gauss points. The resulting sparsity pattern is precomputed by merging the interaction lists of each element vertex, reaching a tremendous benefit in terms of complexity reduction. However, the reported results still exhibit the indexing phase as a possible bottleneck.

A more effective approach, namely the node-pair-wise formulation, has been proposed in [38] for the initialization of the EFGM. A preliminary step is responsible for identifying all the interacting nodes and their shared Gauss points. Moreover, the work exploits geometrical considerations regarding the domain of influences, to minimize the effort of finding the node pairs with at least one shared Gauss point. In fact, Gauss nodes at the frontier of a given node $i$ domain of influence are able to span all the interacting node pairs involving the node $i$.

Algorithm 3 summarizes the main methodologies proposed in the literature for the formulation of the mass and stiffness matrices. The Gauss point-wise approach, relies on a loop over all the quadrature points, each of which contributes to the global matrices with each possible pair of nodes definable within its support area. However, it suffers dramatically the scattered write access required to update the proper entry of the matrices: for each quadrature point, the affected location is stored in non contiguous memory location. In view of parallelism, race conditions due to multiple Gauss points trying to update the same node-pair simultaneously may be prevented.

As discussed in [38], the node-pair-wise approach partially dissolves those problems rearranging the computations such to obtain a gather parallelism: each non-zero entry is computed at once, without any successive update. However, this is achieved at the expenses of computing the lists of the shared Gauss points, $\mathcal{D}_{i j}=\mathcal{D}_{i} \cup \mathcal{D}_{j}$, for each node pair. Besides, a more regular access to memory is achieved for writing the results, at the price of a less regular access in reading from the required input lists.

Summarizing, all the mentioned formulations for mass and stiffness matrices are organized in two steps: some kind of initialization step is required for recovering, and possibly regularizing, the sparsity pattern of the computations, whereas the second stage is responsible for actually filling the matrices, with both steps being computationally involved.

A less demanding alternative is given by the interactionwise approach, which relies only on the knowledge of both primal and dual lists such that the matrices can be formulated reusing information already extracted during the computation of nears and basis functions. Moreover, each row of the matrices can be formulated independently. However, this approach still demands for a scattered and multiple update of the elements of each row, which jeopardizes 
an effective massive parallel execution: a specialized indexing procedure may be needed for gradually populating each row, directly using the sparse formalism. Alternatively, the formulation of the rows should rely on full sizes, leading to excessive memory requirements.

Based on these considerations, this work proposes a cluster-wise approach, detailed in Algorythm 4, which is a variation of the interaction-wise approach, that dissolves the above mentioned memory access related challenges, while also maintaining the advantage of avoiding any precomputation of the resulting sparsity pattern. In fact, it relies merely on pre-computing the Clustered Primal lists and the Dual Lists. A first level loop spans all the clusters of the domain: for each cluster it aims at filling the subset of rows corresponding to all its nodes. The size of these sub-matrices is known from the cardinality of the clusters and the corresponding clustered Primal list, used respectively as the number of rows and columns. The cluster sub-matrices are used during the filling procedure and

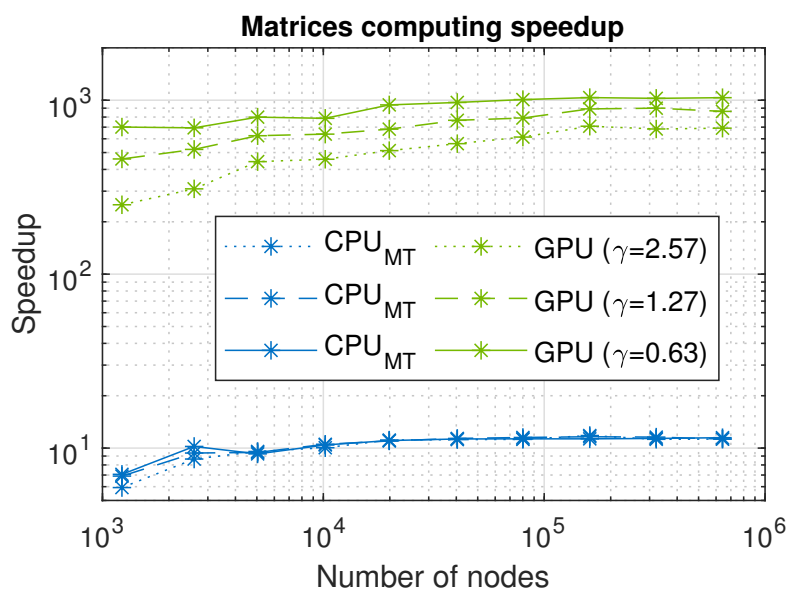

Fig. 11. Matrix Computing: $C P U_{M T}$ and $G P U$ speedup, relative to $C P U_{S T}$, for different values of the $\gamma$ parameter, and the dunavant rule equal to 6 .

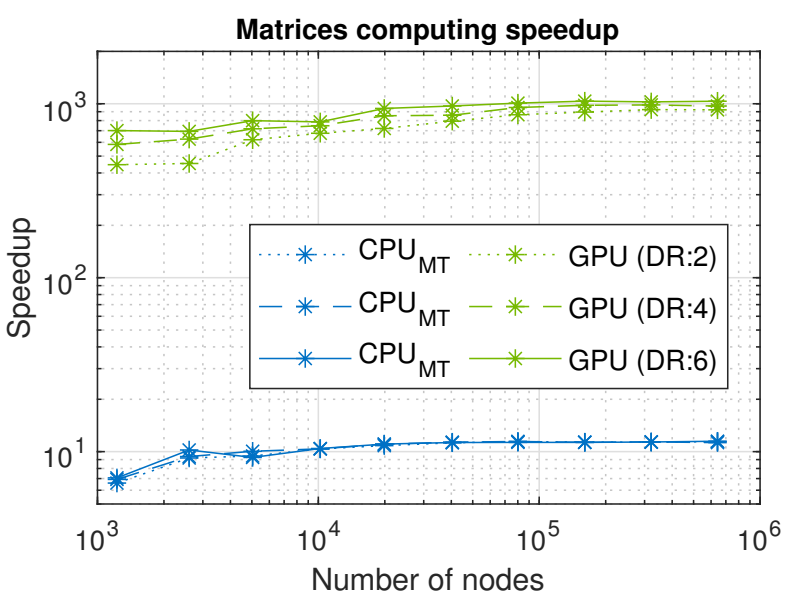

Fig. 12. Matrix Computing: $C P U_{M T}$ and $G P U$ speedup, relative to $C P U_{S T}$, for different values of the dunavant rule, and $\gamma=0.63$. translated to the COO format only at the end of the operation.

The execution on GPU relies on several parallelization paradigms: each cluster is assigned to a different stream, such that two separated kernel launches are used, one for filling the matrices and one for translating to the COO format. Within each kernel, the grid is sized such to have one block for each node potentially interacting with the current cluster, whereas the blocks are sized to have one thread for each node in the cluster. All the block threads are orchestrated to span through all the quadrature points extracted from the Dual List. For each quadrature point, $g$, interacting with the node $j$, each thread of the block will accumulate locally the results corresponding to the nodepair $i-j$. This allows us to exploit the more efficient memory for computing the matrix values, relying on the slower global memory only for saving the partial results to the following kernel. The second kernel launch is queued in the cluster stream, and it is used to transform the kernel matrices to the COO format. The parallel version of the CPU program is obtained by splitting the works at the level of the outer loop, thus each thread works on its own cluster.

Table 5 summarizes the performance results of the proposed algorithm. In particular, for the more demanding example the GPU implementation achieves a speedup of nearly 1000x, showing a slightly increasing trend for bigger support sizes (Fig. 11), but no influence with respect to the integration rule (Fig. 12). In opposite, the CPU saturate to a speedup of $10 \mathrm{x}$, with no influences either from $\gamma$ nor the quadrature rule.

Although the theoretical throughput performances for single precision operations of the different platforms under investigation are:

- Intel XEON E5-2640-v4: $3400 \mathrm{MHz}$ x 1 cores x 32 $\mathrm{IPC}=108.8$ GFLOPs (single thread), 1.09 TFLOPs (multi-thread)

- Nvidia TITAN X : $1531 \mathrm{MHz}$ x 3584 cores x 2 IPC $=10.97$ TFLOPs

the obtained level of speedup is justified considering that the tasks involved in the matrix computations consist mostly of memory-bound operations, where memory latency is the main bottleneck. In facts, the proposed GPU implementation not only benefits from the higher computational throughput, but also from the peculiar capability of GPUs to hide the instruction latency by pipe-lining a huge number of similar computations, and more importantly from the data-localized structure of the algorithm, which allows us to maximize the use of more localized memory, such as cache and registers, for which GPUs offer notably higher performances.

In particular, we conducted a dedicated profiling campaign using the NVIDIA Visual Profiler tool, which is part of the CUDA toolkit: according to the obtained results, the reported GPU performances are achieved while 
Table 5

Computing time in seconds for filling the localized matrices. Speedups, relative to $C P U_{S T}$, are reported in parenthesis.

\begin{tabular}{|c|c|c|c|c|c|c|c|c|c|}
\hline \multirow[b]{2}{*}{ Example } & \multicolumn{3}{|c|}{$\gamma=2.57$} & \multicolumn{3}{|c|}{$\gamma=1.27$} & \multicolumn{3}{|c|}{$\gamma=0.63$} \\
\hline & $C P U_{S T}$ & $C P U_{M T}$ & $G P U$ & $C P U_{S T}$ & $C P U_{M T}$ & $G P U$ & $C P U_{S T}$ & $C P U_{M T}$ & $G P U$ \\
\hline$E 101.2$ & 2.31 & $0.25 \quad(9.2 x)$ & $0.01(231 x)$ & 8.35 & $0.84 \quad(9.9 x)$ & $0.02(440 x)$ & 30.4 & $2.87(10.6 x)$ & $0.04(676 x)$ \\
\hline E101.4 & 4.81 & $0.49 \quad(9.8 x)$ & $0.01(370 x)$ & 16.6 & $1.60(10.4 x)$ & $0.03(574 x)$ & 59.1 & $5.67(10.4 x)$ & $0.08(747 x)$ \\
\hline$E 101.6$ & 10.1 & $0.99(10.2 x)$ & $0.02(458 x)$ & 33.8 & $3.29(10.3 x)$ & $0.05(638 x)$ & 118. & $11.3(10.5 x)$ & $0.15(787 x)$ \\
\hline E201.2 & 10.1 & $0.95(10.7 x)$ & $0.03(327 x)$ & 35.5 & $3.37(10.5 x)$ & $0.06(563 x)$ & 129. & $11.6(11.1 x)$ & $0.16(794 x)$ \\
\hline$E 201.4$ & 21.6 & $1.96(11.0 x)$ & $0.04(481 x)$ & 70.6 & $6.43(11.0 x)$ & $0.11(660 x)$ & 253. & $23.3(10.9 x)$ & $0.29(859 x)$ \\
\hline$E 201.6$ & 42.2 & $3.87(10.9 x)$ & $0.07(563 x)$ & 143. & $12.6(11.3 x)$ & $0.19(766 x)$ & 501. & $45.4(11.0 x)$ & $0.52(971 x)$ \\
\hline$E 401.2$ & 39.8 & $3.72(10.7 x)$ & $0.11(358 x)$ & 144. & $12.9(11.2 x)$ & $0.23(620 x)$ & 529. & $47.0(11.3 x)$ & $0.59(896 x)$ \\
\hline$E 401.4$ & 87.4 & $7.83(11.2 x)$ & $0.17(514 x)$ & 301. & $26.0(11.6 x)$ & $0.39(769 x)$ & 1037. & $94.1(11.0 x)$ & $1.06(980 x)$ \\
\hline$E 401.6$ & 178. & $15.4(11.6 x)$ & $0.25(709 x)$ & 593. & $51.8(11.4 x)$ & $0.67(891 x)$ & 2070. & 186. $(11.1 x)$ & $2.00(1035 x)$ \\
\hline$E 801.2$ & 156. & $13.8(11.3 x)$ & $0.42(372 x)$ & 575. & $52.2(11.0 x)$ & $0.80(718 x)$ & 2067. & 185. $(11.1 x)$ & $2.24(922 x)$ \\
\hline$E 801.4$ & 326. & $29.7(11.0 x)$ & $0.56(587 x)$ & 1142. & $99.7(11.5 x)$ & $1.38(825 x)$ & 4088. & 368. $(11.1 x)$ & $4.21(970 x)$ \\
\hline$E 801.6$ & 637. & $57.0(11.2 x)$ & $0.92(691 x)$ & 2244. & 201. (11.1x) & $2.60(862 x)$ & 8252. & 722. $(11.4 x)$ & $7.98(1034 x)$ \\
\hline
\end{tabular}

reaching around $55 \%$ of the available shared memory bandwidth and $76 \%$ of the total compute throughput divided as follows: $45 \%$ of arithmetic operations, $20 \%$ of memory operations, and the remaining $11 \%$ of control-flow operations. More in detail, a level of utilization for the shared memory load operations of about $3400 \mathrm{~GB} / \mathrm{s}$ is achieved. Given that the reported GPU-CPU speedups are one order of magnitude bigger than the expected levels, we may conclude that the current CPU implementations do not achieve a comparable level of quality with respect to the GPU, owing to a poor utilization of the cache mechanisms.

\subsection{Effects of the non-dimensional parameter, $\gamma$}

As discussed in Section 2, the computational complexity related to LME schemes initialization is deeply influenced from the non-dimensional shape parameter $\gamma$. Therefore, this section considers more in detail the impact of $\gamma$ on the speed-up of the proposed algorithms. In particular, we analyze increasing levels of $\gamma$, in the range between 0.2 and 3.0, for a unit square domain having 40000 nodes, and about half million quadrature points. For each test, we use a cluster size target of 1024 points.

The speedup results are reported in Fig. 13. While the CPU versions do not show any particular trend related to $\gamma$, the GPU implementations tend to show an increasingly better speedup, when increasing the support size areas. In addition, for the the basis functions computation reported in Fig. 13a, we notice different performance peaks, which we explain considering that for each peak, the support areas of each quadrature point is big enough to influence a number of nodes which is a multiple of the warp size, which corresponds to the number of threads actually executed in parallel, on each streaming multiprocessor of the GPU.

\subsection{Effects of the cluster size}

The influence of the cluster size on the performance of the proposed algorithm is also considered more in detail in this section, where we analyze increasing cluster sizes, ranging from 16 to 1024 nodes-per-cluster targets, considering a square unit domain discretized again with 40401 nodes and a shape parameter $\gamma=1.27$.

The corresponding speedup results are reported in Fig. 14. For the nears computing phase (Fig. 14a), we notice a strong impact on the scalability of the CPU implementation, which achieves the best performance only for the largest cluster sizes, whereas no particular trend is noted for the GPU implementations.

For the matrix formulation phase (Fig. 14c), we notice a deterioration of the CPU performance for very large cluster sizes, which can be due to the fact that larger sizes compromise the exploitation of some levels of CPU cache. In fact, when the size of each cluster is too big to not fit into the cache, a less efficient memory access takes place, with a considerably worse performance result. In opposite, GPU implementations show a more stable trend, with a first peak performance around a cluster size of $64(=8 \times 8)$ nodes, followed by another, and slightly better peak, around 256 $(=16 \times 16)$ nodes. It is also interesting to note that for very small cluster, the GPU is under-exploiting its potential due to not optimal occupancy. In facts, each streaming multiprocessor (SM) of the GPU is able to execute at least two warps of thread simultaneously (1warp $=32$ threads), which in this case leads to have optimal speedups for size of the clusters big enough to saturate the SMs.

\subsection{Effects of the number of threads on the CPU workload}

This section analyzes the scalability of the CPU implementations, i.e. the impact of using an increasing number of threads for executing the proposed algorithm on the CPU. The analysis is performed considering a square unit domain discretized with 40401 nodes and a shape parameter $\gamma=0.63$, and a nodes-per-cluster target of 64 nodes. Two different CPU configurations are analyzed: the 10cores Intel XEON E5-2640-v4 machine of the previous ex- 


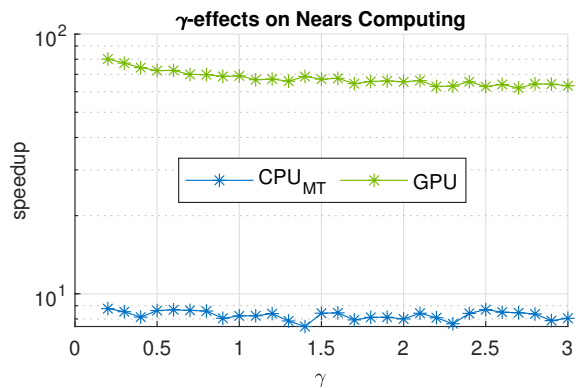

(a)

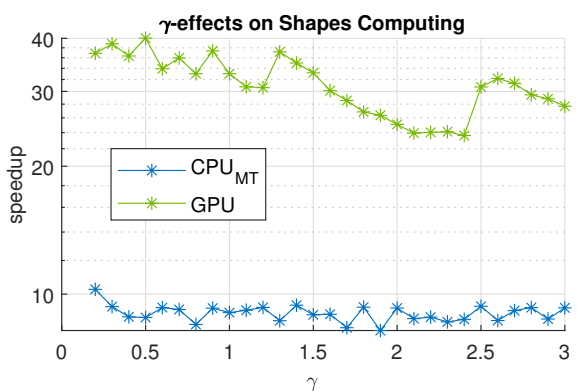

(b)

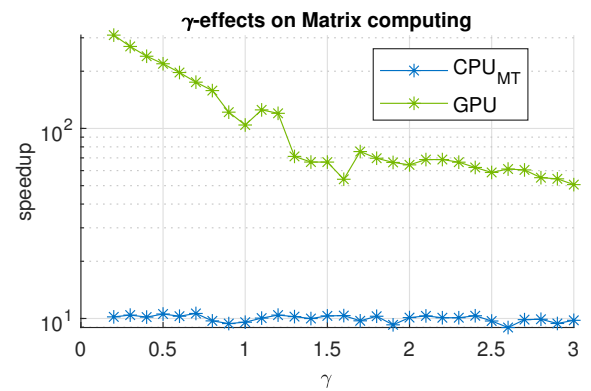

(c)

Fig. 13. Effects of the shape parameter $\gamma$ on the efficiency of computing (a) the nears, (b) the basis functions and (c) the mass and stiffness matrices.

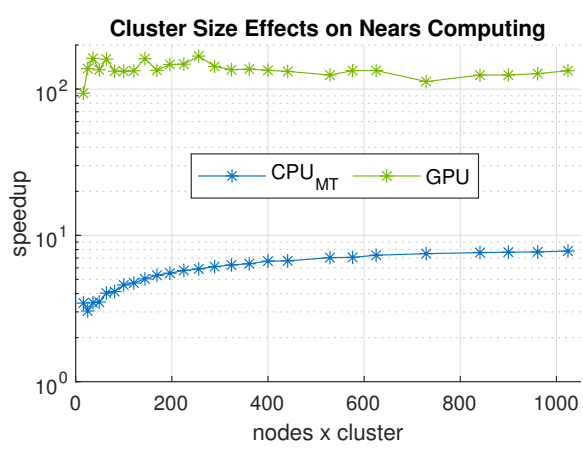

(a)

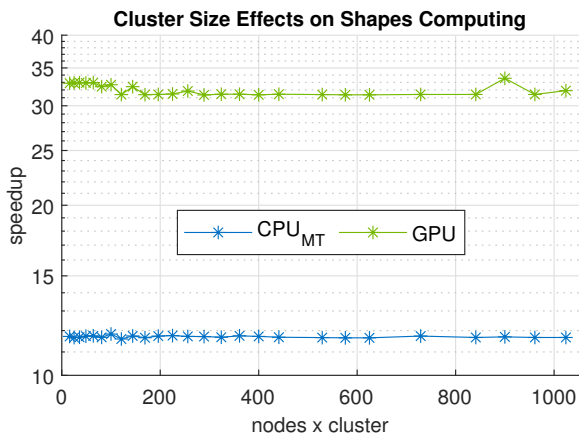

(b)

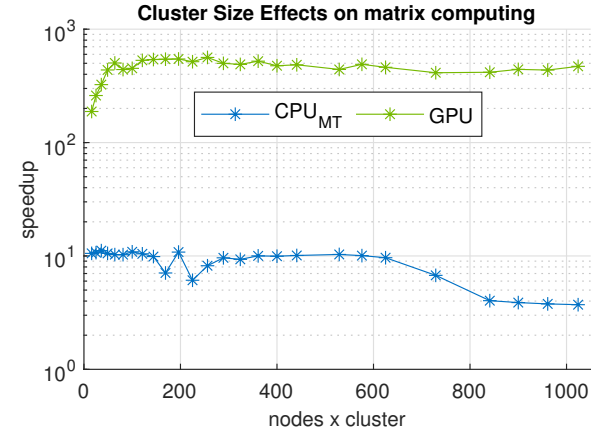

(c)

Fig. 14. Effects of the cluster size on the efficiency of computing (a) the nears, (b) the basis functions and (c) the mass and stiffness matrices.

amples, and a 4-cores Intel i7-4710MQ which is also used later for the numerical examples in Section 4 . Both CPUs are used with Intel(R) Hyper Threading functionality enabled. The number of threads used to split the parallel section of the algorithms is varied from 1 to 20 for the 4cores CPU, and 1-28 for the 10-core CPU.

Fig. 15 summarizes the obtained speedups, computed by selecting the best computational execution times out of 10 repetitions. Due to Atomic operations, a sub-linear scalability is reported for the Nears Computing (Fig. 15(a)), whereas an almost perfect scaling is achieved for both the Shape Computing (Fig. 15(b)) and the Matrix Computing (Fig. 15(c)) stages. As expected, for all the stages, and for both the considered platforms, a first reduction of the scalability is reported when the number of thread exceeds the number of cores, with a second peak achieved when using two threads for each available core and thus obtaining the maximum benefit from hyper-threading.

It is worth noting that for the Nears and the Shapes computing stage, the performance tends to worsen again after the achieved best performance, while for the Matrix Computing we observe a more stable asymptotic trend. More importantly, our analysis showed that thanks to hyperthreading the best $C P U_{M T}$ performances are achieved when using a number of thread equal to the number of logical cores, which for the used machines correspond to twice the physical cores.

\section{Numerical Examples}

The performance of the initialization procedures presented in Section 3 is further investigated in this section considering two numerical examples, both governed by the Helmholtz equation (12). The first case-study deals with the vibrations of a rectangular membrane, fixed at its boundaries, and compares the results with the theoretical solution, whereas the second case study focus on the acoustic wave propagation within a car cavity. To verify the usefulness of the proposed methodology on commodity hardware, both problems are simulated on a Dell Precision M6800 laptop featuring a Nvidia Quadro K2200 video-card, an Intel i7-4710MQ 2.5 GHz, and 32 GB RAM.

\subsection{D membrane vibrations}

The natural vibrations of a rectangular membrane, with size $a=1.3 \mathrm{~m}$ and $b=1.3 \mathrm{~m}$, are computed by solving the eigenvalue problem associated to Eq. 12, with essential boundary conditions $(u=0$ on $\partial \Omega)$. The analytical solution for the eigenmodes and the corresponding eigen- 


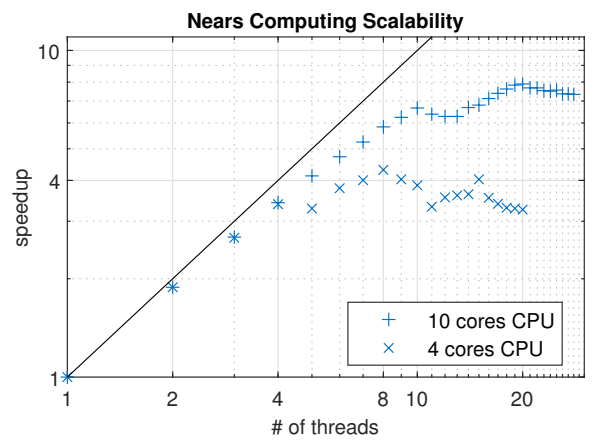

(a)

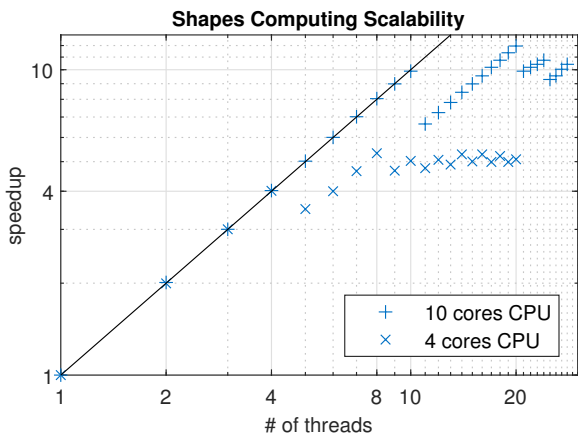

(b)

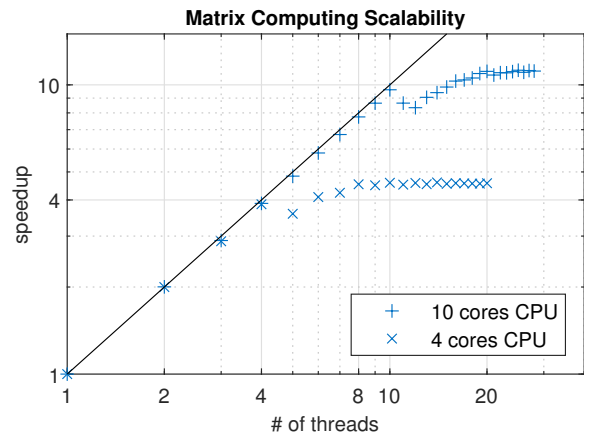

(c)

Fig. 15. Effects of the number of threads on the CPU multi-thread scalability of computing (a) the nears, (b) the basis functions and (c) the mass and stiffness matrices.

\section{Table 6}

Computing performances for two mesh size of the vibrating rectangular membrane.

\begin{tabular}{rrrrrrr}
\hline & \multicolumn{3}{c}{$\gamma=0.63, h=0.03$} & \multicolumn{3}{c}{$\gamma=0.63, h=0.01$} \\
Nodes & \multicolumn{3}{c}{1921} & & & \\
& 2486768 & \\
Gauss Points & \multicolumn{3}{c}{2496648} & \\
Execution Times[s] & $C P U_{S T}$ & $C P U_{M T}$ & $G P U$ & $C P U_{S T}$ & $C P U_{M T}$ & $G P U$ \\
\hline Nears Computing & 0.052 & 0.023 & 0.004 & 2.0 & 0.77 & 0.070 \\
Shapes Computing & 0.082 & 0.017 & 0.034 & 2.6 & 0.54 & 0.671 \\
Matrix Computing & 2.954 & 0.482 & 0.040 & 462 & 50.7 & 4.381 \\
\hline Total time & 3.088 & 0.522 & 0.078 & 467 & 52.0 & 5.122 \\
Speedup & & $6 \mathrm{x}$ & $40 \mathrm{x}$ & & $9 \mathrm{x}$ & $91 \mathrm{x}$
\end{tabular}

frequencies is given by the following relations:

$$
\begin{aligned}
\Phi_{(m, n)}(x, y) & =\sin \frac{m \pi x}{a} \sin \frac{n \pi y}{b}, \quad \begin{array}{l}
m=1,2, \ldots \\
n=1,2, \ldots
\end{array} \\
\omega_{(m, n)} & =c \pi \sqrt{\frac{m^{2}}{a^{2}}+\frac{n^{2}}{b^{2}}} \quad,
\end{aligned}
$$

where $c$ is the speed of sound.

As outlined in Fig. 16, the rectangular domain is meshed

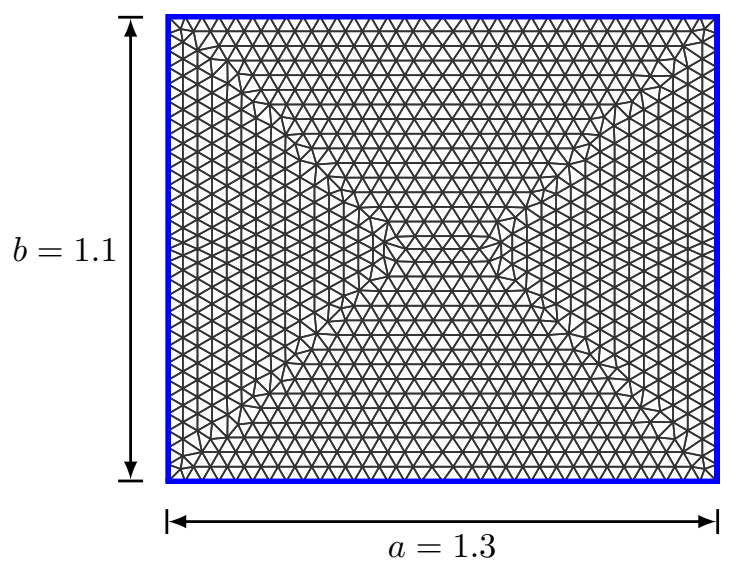

Fig. 16. A mesh with a characteristic length $h=0.03$ used for the simulation of the natural vibrations of a rectangular membrane. using the MATLAB tool Mesh2d [49], which provides a slightly more irregular nodal distribution with respect to the tensor product grids employed in the previous section. Two mesh sizes are considered : $h=0.03$ and $h=0.01$, for LME approximants computed with $\gamma=0.63$, and a second order integration rule is used.

A set of 15 modes, computed by the numerical simulation and the associated relative error on the frequency are outlined in Fig. 17, while the performance of the proposed algorithms are compared and summarized in Table 6. As expected, the GPU-accelerated approach allows the computation of the matrices in a few seconds, even with a relatively higher mesh density, which is crucial to increase the accuracy for high scale dynamic applications.

\subsection{Acoustic field inside a car cavity}

This second examples considers a more relevant industrial application, consisting in the computation of the acoustic field inside a vehicle, which is represented by the two-dimensional model outlined in Fig. 19. The domain is meshed again with Mesh2d for a caractersitic lenght $h=0.01$, which results in a triangulation of 14705 nodes and 28510 triangles. The eigenvalue problem associated to the Helmholtz equation is solved in this case with natural boundary conditions $(\nabla u \cdot \mathbf{n}=0$ on $\partial \Omega)$ using LME approximants computed with $\gamma=0.63$ and a second order integration rule. The first 3 modes with non zero frequency are outlined in Fig. 18. 


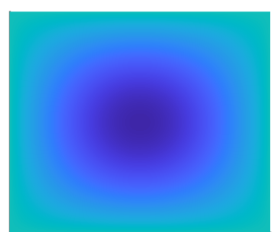

$(1,1): 6.82 \mathrm{e}-04$

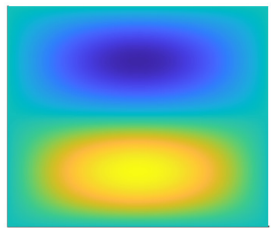

$(2,1): 2.47 \mathrm{e}-04$

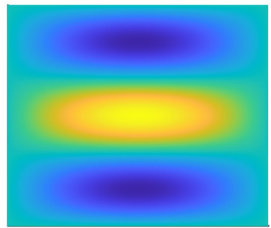

$(3,1): 1.19 \mathrm{e}-04$

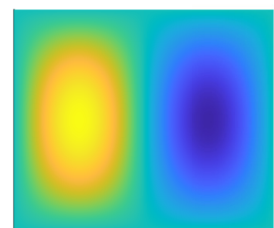

$(1,2): 3.01 \mathrm{e}-04$

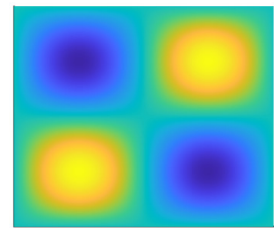

$(2,2): 1.68 \mathrm{e}-04$

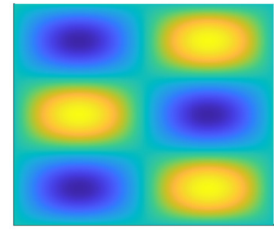

$(3,2): 9.66 e-05$

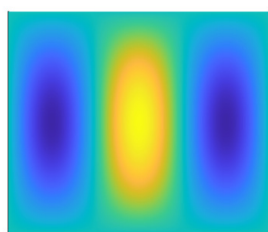

$(1,3): 1.55 e-04$

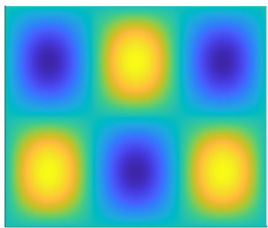

$(2,3): 1.10 e-04$

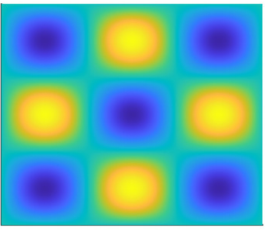

$(3,3): 7.38 \mathrm{e}-05$

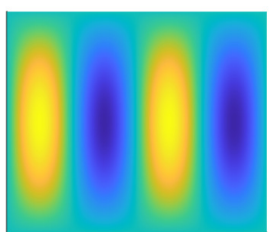

$(1,4): 9.21 \mathrm{e}-05$

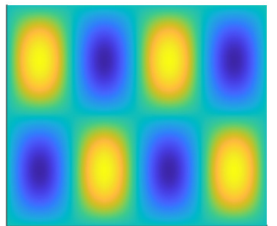

$(2,4): 7.36 \mathrm{e}-05$

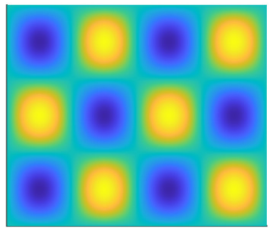

$(3,4): 5.53 \mathrm{e}-05$

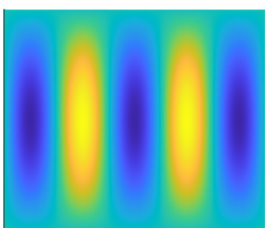

$(1,5): 6.01 \mathrm{e}-05$

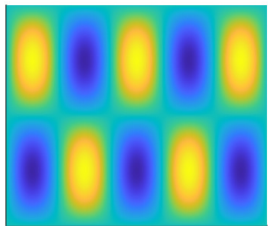

$(2,5): 5.15 e-05$

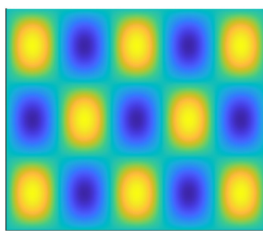

$(3,5): 4.15 e-05$

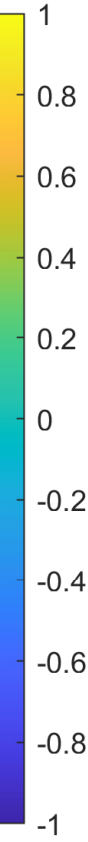

0.8

0.6

0.4

0.2

$-0.2$

$-0.4$

$-0.6$

$-0.8$

Fig. 17. The first 15 eigenmodes with non-zero frequency describing the natural vibrations of rectangular membrane. For each mode, the relative error of the associated frequency with respect to the analytical value is reported.

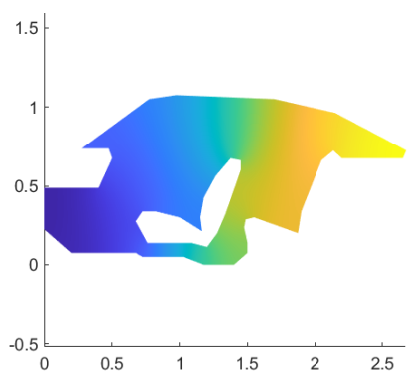

(a)

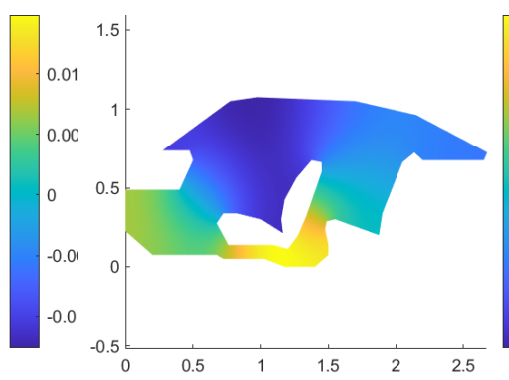

(b)

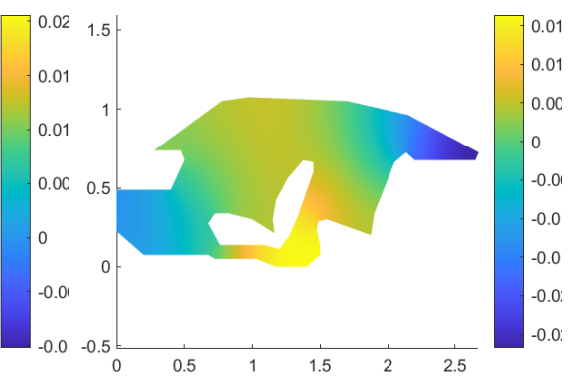

(c)

Fig. 18. The first 3 eigenmodes with non-zero frequency associated to the Helmholtz problem on the car cavity domain.

The computational times associated with the matrix assembly procedure are reported in Table 7 , where it can be observed how the total computing time for the GPU implementation is in the order of a few seconds, with a significant speedup with respect to the standard CPU one. Remarkably, this example verifies the validity of the proposed clustering algorithm also for the particular car cavity topology, with a more irregular domain and a hole.

\section{Table 7}

Computational times for the car cavity example.

\begin{tabular}{r|ccc}
\hline Execution Time[ms] & $C P U_{S T}$ & $C P U_{M T}$ & $G P U$ \\
\hline Nears Computing & 0.8 & 0.4 & 0.061 \\
Shapes Computing & 2.4 & 0.5 & 0.577 \\
Matrix Computing & 110 & 28.7 & 2.398 \\
\hline Total & 113 & 29.6 & 3.036 \\
Speedup & & $3.8 \mathrm{x}$ & $37 \mathrm{x}$
\end{tabular}

\section{Conclusions}

In this paper, we propose a cluster-wise approach for an effective initialization of LME meshfree methods, where we combine the advantages of regioning discussed in [38] with the benefits of locally aggregating the interaction lists, as proposed in [23]. Our approach obtains an advantageous minimization of the pre-processing stages, typically required for the formulation of the sparsity pattern. As listed in Algorythm 4 for each cluster $c$, we retrieve the aggregated Primal list, $\mathcal{C} \mathcal{P}_{c}$, i.e. the collection of all the nodes potentially interacting with at least one of the node of the cluster, exploiting only the geometrical aspects of the problem. Similarly to [23], the proposed formulation accumulates the results of each cluster using intermediate full storage representation, maintaining also the massive parallel amenable for the computation of each element [38]. The matrices, which share the same sparsity pattern, are 


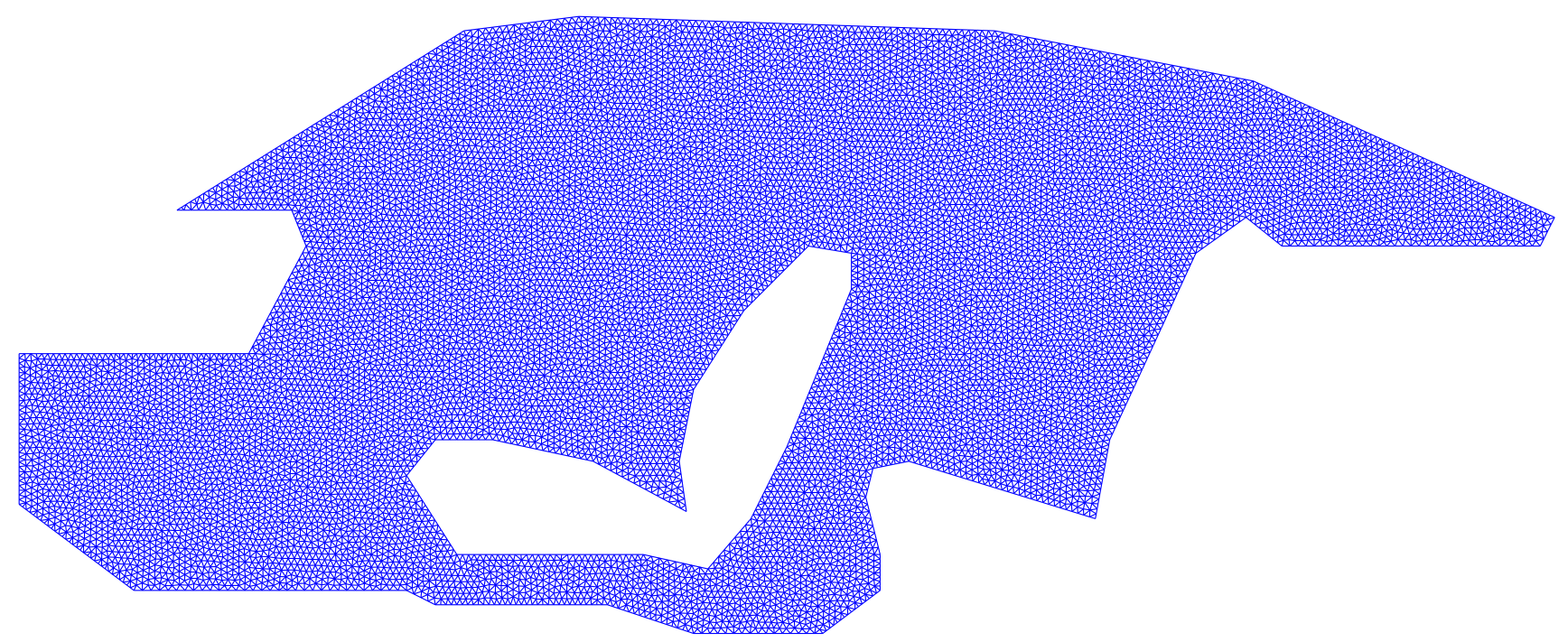

Fig. 19. A two dimensional representation of the acoustic cavity inside a vehicle and the corresponding mesh used for the problem discretization and integration with 14705 nodes.

transformed to the chosen sparse format only when they are assembled into the global structure.

Numerical results shows that the proposed approach is amenable for massive parallel acceleration in both CPU and GPU architectures. In particular, industrially relevant problems sizes, constituted by hundreds of thousands nodes can be solved in a modern workstation in a matter of seconds, thus enabling the usage of LME approximants for the solution of vibrational and acoustic problems, where this type of approximation introduces some computational advantages with respect to the classical FEM [25].

Further developments of the presented work can consider the possibility of restructuring the computational flow, in order to optimally balance the computational efforts between CPU and GPU, and eventually minimize the global memory footprint. Besides, an extension to three dimensions is also planned. In this case, the number of neighbors for each evaluation point drastically increases and significantly higher computational times are expected for all the implementations, reflecting the wellknown curse of dimensionality phenomenon. However, observing that a computationally similar effect is obtained by lowering the $\gamma$ parameter, we expect the 3D speedups of the GPU implementation to be similar or even better, depending on the computational stage, than those reported in Section 3.5 for the $2 \mathrm{D}$ case. As possible application examples, the developed methodology may pave the way to LME meshfree adoption for simulation of modern multimaterial specimens, where material properties such as stiffness, density, and orthotropicity may vary at a finer level.

Further works may be dedicated to optimize the memory access patterns also on the CPU, circumventing the poor cache memory performances at the root of the poor CPU multi-thread performance. Moreover, further developments may try to extend the multi-threaded execution to a more advanced parallel paradigms exploiting also the instruction set extensions of the processor, such as the Streaming SIMD Extension (SSE) or the Advanced Vector Extension (AVE).

\section{Acknowledgments}

The authors gratefully acknowledge the support of the European Commission: the research of Francesco Cosco is funded by the European Commission - FSE and FESR, within the PON R\& I 2014-2020 programme, Action I.2 Attraction and International Mobility (AIM1857122); the research of F. Greco has received funding from the European Union's Horizon 2020 research and innovation programme, under the Marie Sklodowska-Curie grant agreement No 792028. The KU Leuven research fund is also gratefully acknowledged for its support.

\section{References}

[1] T. Belytschko, Y. Krongauz, D. Organ, M. Fleming, and P. Krysl, "Meshless methods: An overview and recent developments," Computer Methods in Applied Mechanics and Engineering, vol. 139, no. 1, pp. 3-47, 1996.

[2] A. Huerta, T. Belytschko, S. Fernández-Méndez, and T. Rabczuk, "Meshfree methods," in Encyclopedia of Computational Mechanics (E. Stein, R. de Borst, and T. Hughes, eds.), vol. 1, ch. 10, pp. 279-309, John Wiley \& Sons, Ltd., 2004.

[3] S. Li and W. K. Liu, "Meshfree and particle methods and their applications," Applied Mechanics Reviews, vol. 55, no. 1, pp. 134, 2002.

[4] J.-S. Chen and T. Belytschko, "Meshless and Meshfree Methods," Encyclopedia of Applied and Computational Mathematics, pp. 886-894, 2015.

[5] T. Belytschko, Y. Y. Lu, and L. Gu, "Element-free Galerkin methods," International journal for numerical methods in engineering, vol. 37, no. 2, pp. 229-256, 1994. 
[6] W. K. Liu, S. Jun, and Y. F. Zhang, "Reproducing kernel particle methods," International journal for numerical methods in fluids, vol. 20, no. 8-9, pp. 1081-1106, 1995.

[7] P. Lancaster and K. Salkauskas, "Surfaces generated by moving least squares methods," Mathematics of Computation, vol. 37, no. 155 , pp. 141-158, 1981.

[8] S. Fernández-Méndez and A. Huerta, "Imposing essential boundary conditions in mesh-free methods," Computer methods in applied mechanics and engineering, vol. 193, no. 12, pp. 1257-1275, 2004.

[9] G.-R. Liu and Y. Gu, "A point interpolation method for twodimensional solids," International Journal for Numerical Methods in Engineering, vol. 50, no. 4, pp. 937-951, 2001.

[10] J. Wang and G. Liu, "A point interpolation meshless method based on radial basis functions," International Journal for $\mathrm{Nu}$ merical Methods in Engineering, vol. 54, no. 11, pp. 1623-1648, 2002 .

[11] N. Sukumar, The natural element method in solid mechanics. PhD thesis, Northwestern University, 1998.

[12] I. Alfaro, J. Yvonnet, F. Chinesta, and E. Cueto, "A study on the performance of natural neighbour-based Galerkin methods," International Journal for Numerical Methods in Engineering, vol. 71 , no. 12 , pp. $1436-1465,2007$.

[13] M. Arroyo and M. Ortiz, "Local maximum-entropy approximation schemes: a seamless bridge between finite elements and meshfree methods," International Journal for Numerical Methods in Engineering, vol. 65, no. 13, pp. 2167-2202, 2006.

[14] E. T. Jaynes, "Information theory and statistical mechanics," Physical review, vol. 106, no. 4, p. 620, 1957.

[15] N. Sukumar, "Construction of polygonal interpolants: a maximum entropy approach," International Journal for Numerical Methods in Engineering, vol. 61, no. 12, pp. 2159-2181, 2004.

[16] A. Ortiz, M. Puso, and N. Sukumar, "Maximum-entropy meshfree method for compressible and near-incompressible elasticity," Computer Methods in Applied Mechanics and Engineering, vol. 199, no. 25, pp. 1859-1871, 2010.

[17] F. Greco, L. Filice, C. Peco, and M. Arroyo, "A stabilized formulation with maximum entropy meshfree approximants for viscoplastic flow simulation in metal forming," International Journal of Material Forming, pp. 1-13, 2014.

[18] D. Millán and M. Arroyo, "Nonlinear manifold learning for model reduction in finite elastodynamics," Computer Methods in Applied Mechanics and Engineering, vol. 261, pp. 118-131, 2013.

[19] C. J. Cyron, K. Nissen, V. Gravemeier, and W. A. Wall, "Stable meshfree methods in fluid mechanics based on green's functions," Computational Mechanics, vol. 46, no. 2, pp. 287-300, 2010.

[20] F. Amiri, D. Millán, Y. Shen, T. Rabczuk, and M. Arroyo, "Phase-field modeling of fracture in linear thin shells," Theoretical and Applied Fracture Mechanics, vol. 69, pp. 102-109, 2014.

[21] B. Hashemian, D. Millán, and M. Arroyo, "Modeling and enhanced sampling of molecular systems with smooth and nonlinear data-driven collective variables," The Journal of chemical physics, vol. 139, no. 21, p. 214101, 2013.

[22] A. Rosolen, C. Peco, and M. Arroyo, "An adaptive meshfree method for phase-field models of biomembranes. part i: Approximation with maximum-entropy basis functions," Journal of Computational Physics, vol. 249, pp. 303-319, 2013.

[23] C. Peco, A. Rosolen, and M. Arroyo, "An adaptive meshfree method for phase-field models of biomembranes. part ii: A Lagrangian approach for membranes in viscous fluids," Journal of Computational Physics, vol. 249, pp. 320-336, 2013.

[24] D. Millán, A. Rosolen, and M. Arroyo, "Thin shell analysis from scattered points with maximum-entropy approximants," International Journal for Numerical Methods in Engineering, vol. 85, no. 6, pp. 723-751, 2011.

[25] F. Greco, L. Coox, and W. Desmet, "Maximum-entropy methods for time-harmonic acoustics," Computer Methods in Applied Mechanics and Engineering, vol. 306, pp. 1-18, 2016.
26] A. Bompadre, B. Schmidt, and M. Ortiz, "Convergence analysis of meshfree approximation schemes," SIAM Journal on Numerical Analysis, vol. 50, no. 3, pp. 1344-1366, 2012.

[27] A. Bompadre, L. Perotti, C. Cyron, and M. Ortiz, "Convergent meshfree approximation schemes of arbitrary order and smoothness," Computer Methods in Applied Mechanics and Engineering, vol. 221, pp. 83-103, 2012.

[28] A. Rosolen and M. Arroyo, "Blending isogeometric analysis and local maximum entropy meshfree approximants," Computer Methods in Applied Mechanics and Engineering, vol. 264, pp. 95-107, 2013.

29] F. Greco, A. Rosolen, L. Coox, and W. Desmet, "Contact mechanics with maximum-entropy meshfree approximants blended with isogeometric analysis on the boundary," Computers and Structures, vol. 182, pp. 165-175, 2017.

[30] F. Greco, L. Coox, F. Maurin, and W. Desmet, "NURBSenhanced maximum-entropy schemes," Computer Methods in Applied Mechanics and Engineering, vol. 317, pp. 580-597, 2017.

[31] C. Peco, D. Millán, A. Rosolen, and M. Arroyo, "Efficient implementation of galerkin meshfree methods for large-scale problems with an emphasis on maximum entropy approximants," Computers \& Structures, vol. 150, pp. 52-62, 2015.

[32] J. Nickolls, I. Buck, M. Garland, and K. Skadron, "Scalable parallel programming with cuda," Queue, vol. 6, pp. 40-53, Mar. 2008.

[33] N. Corp., "Nvidia cuda. compute unified device architecture," June 2007.

[34] M. Papadrakakis, G. Stavroulakis, and A. Karatarakis, "A new era in scientific computing: Domain decomposition methods in hybrid cpu-gpu architectures," Computer Methods in Applied Mechanics and Engineering, vol. 200, no. 13-16, pp. 1490 1508, 2011.

[35] A. R. Brodtkorb, T. R. Hagen, and M. L. Sætra, "Graphics processing unit (gpu) programming strategies and trends in \{GPU computing," Journal of Parallel and Distributed Computing, vol. 73 , no. 1 , pp. $4-13,2013$. Metaheuristics on \{GPUs\}.

[36] A. C. Crespo, J. M. Dominguez, A. Barreiro, M. GómezGesteira, and B. D. Rogers, "GPUs, a new tool of acceleration in CFD: Efficiency and reliability on smoothed particle hydrodynamics methods," PLOS ONE, vol. 6, p. e20685, jun 2011

37] G. Kosec and P. Zinterhof, "Local strong form meshless method on multiple Graphics Processing Units," CMES - Computer Modeling in Engineering and Sciences, vol. 91, no. 5, pp. 377396, 2013.

[38] A. Karatarakis, P. Metsis, and M. Papadrakakis, "Gpuacceleration of stiffness matrix calculation and efficient initialization of efg meshless methods," Computer Methods in Applied Mechanics and Engineering, vol. 258, pp. 63-80, 2013.

[39] A. Karatarakis, P. Karakitsios, and M. Papadrakakis, "Gpu accelerated computation of the isogeometric analysis stiffness matrix," Computer Methods in Applied Mechanics and Engineering, vol. 269, pp. 334-355, 2014.

[40] C. E. Shannon, "A mathematical theory of communication," Bell system technical journal, vol. 27, 1948.

[41] V. Rajan, "Optimality of the Delaunay triangulation in $R^{d}$," Discrete $\&$ Computational Geometry, vol. 12, no. 1, pp. 189202, 1994.

[42] F. Greco and N. Sukumar, "Derivatives of maximum-entropy basis functions on the boundary: Theory and computations," International Journal for Numerical Methods in Engineering, vol. 94 , no. 12 , pp. 1123-1149, 2013.

[43] N. Sukumar, "Fortran 90 library for maximum-entropy basis functions. user's reference manual version 1.4. code available at http://www.imechanica.org/node/3424," 2008.

[44] D. Dunavant, "High degree efficient symmetrical gaussian quadrature rules for the triangle," International journal for numerical methods in engineering, vol. 21, no. 6, pp. 1129-1148, 1985.

[45] M. Dendaluce Jahnke, F. Cosco, R. Novickis, J. Perez Rastelli, and V. Gomez-Garay, "Efficient neural network implemen- 
tations on parallel embedded platforms applied to real-time torque-vectoring optimization using predictions for multi-motor electric vehicles," Electronics, vol. 8, no. 2, p. 250, 2019.

[46] S. Arya, D. M. Mount, N. S. Netanyahu, R. Silverman, and A. Y. $\mathrm{Wu}$, "An optimal algorithm for approximate nearest neighbor searching fixed dimensions," Journal of the ACM (JACM), vol. 45, no. 6, pp. 891-923, 1998.

[47] F. Ihlenburg, Finite element analysis of acoustic scattering, vol. 132. Springer Science \& Business Media, 2006.

[48] W. Desmet and D. Vandepitte, "Finite element method in acoustics," in Proceedings of the International Seminar on Applied Acoustics, 1999.

[49] D. Engwirda, "Unstructured mesh methods for the navierstokes equations," Undergraduate Thesis, School of Engineering, University of Sidney, 2005. 